\title{
Electrogenerated Chemiluminescence of A Series of Donor-Acceptor Molecules and X-ray Crystallographic Evidence for the Reaction Mechanisms
}

\author{
Xiao Jiang, ${ }^{\dagger}$ Xichuan Yang, ${ }^{* \dagger}$ Changzhi Zhao, ${ }^{\ddagger}$ Kun Jin, ${ }^{\dagger}$ and Licheng Sun ${ }^{*},, \S$
}

State Key Laboratory of Fine Chemicals, DUT-KTH Joint Education and Research Center on Molecular Devices, Dalian University of Technology(DUT), 158 Zhongshan Rd. 116012 Dalian, China, College of Chemistry and Molecular Engineering, Qingdao University of Science and Technology, 53 Zhengzhou Rd. 266042 Qingdao, China, and KTH School of Chemical Science and Engineering, Organic Chemistry, Teknikringen 30, 10044 Stockholm, Sweden

\section{Contents}

1. Synthetic methodology for the preparation of intermediates $\mathbf{A}, \mathbf{B}, \mathbf{C}$, and compounds $\mathbf{1 - 3}$.

2. ${ }^{1} \mathrm{H}$ NMR and ${ }^{13} \mathrm{C}$ NMR spectra of compounds $\mathbf{1 - 3}$ and intermediates $\mathbf{A}, \mathbf{B}$.

3. Electrochemistry of compounds 1-3.

4. Electrogenerated chemiluminescence of compounds 1-3.

5. Fluorescence and absorption spectra of compound 3a with different concentrations in 1:1 $\mathrm{PhH} / \mathrm{MeCN}$ solution.

1. Synthetic methodology for the preparation of intermediates A, B, C, and compounds 1-3. 

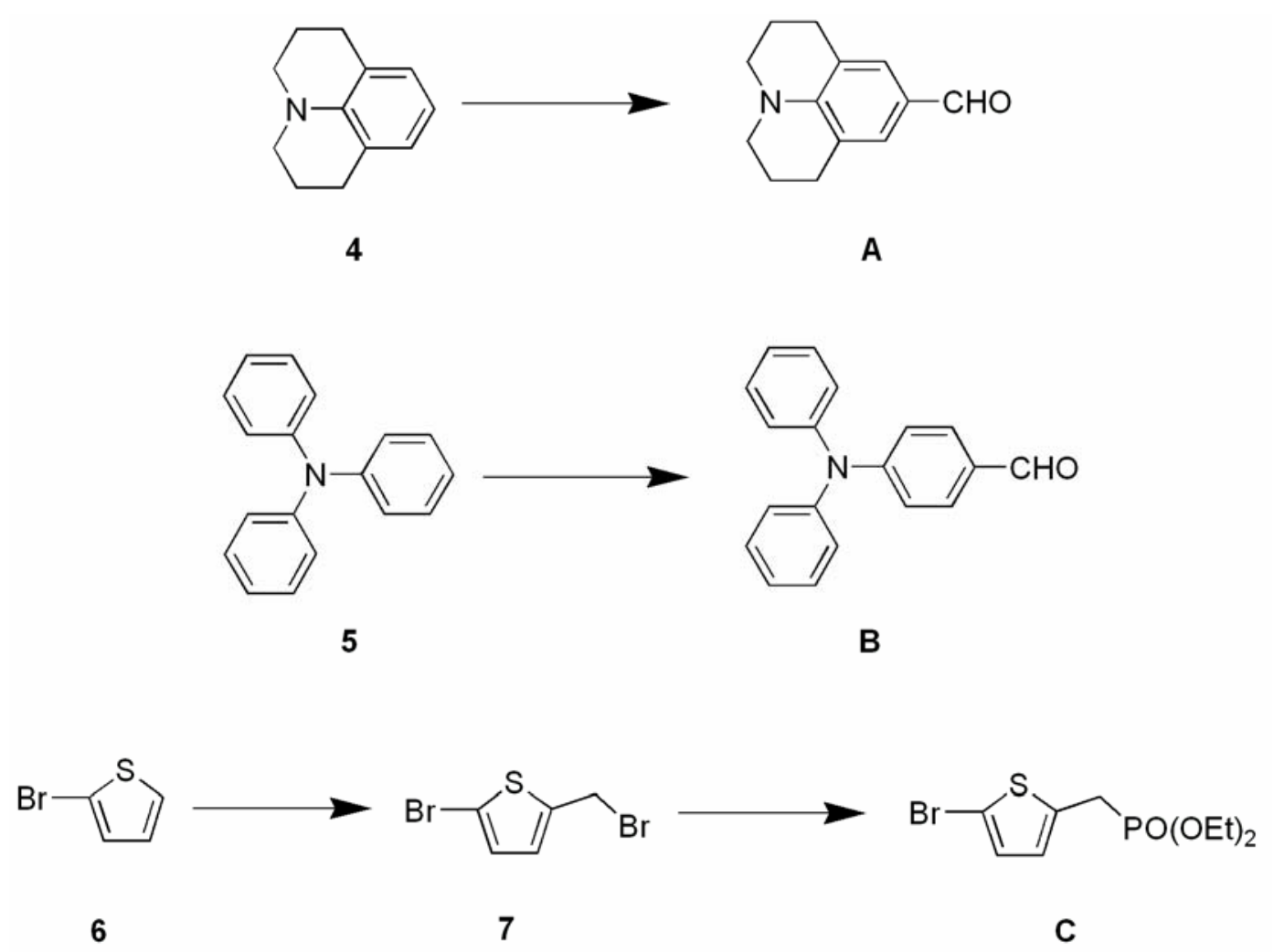

Scheme 1. The synthesis of the intermediates $\mathbf{A}, \mathbf{B}$ and $\mathbf{C}$

\section{General procedure for the synthesis of 2,3,6,7-tetrahydro- $1 \mathrm{H}, 5 \mathrm{H}$-pyrido[3,2,1-ij]quinoline-9-}

carbaldehyde (A). Anhydrous DMF (10 ml) was placed in a two-necked $100 \mathrm{ml}$ flask and cooled in an ice bath. Phosphorous oxychloride (2.65 g, $17.3 \mathrm{mmol}$ ) was then added, dropwise, with stirring. After 30 min, a solution of compound 4 ( $3.0 \mathrm{~g}, 17.3 \mathrm{mmol})$ in DMF (10 ml) was added. The solution was heated at $90{ }^{\circ} \mathrm{C}$ for $3 \mathrm{~h}$. The reaction mixture was then cooled, poured into crushed ice in a beaker, and neutralized to $\mathrm{pH}$ 6-8 by an addition of saturated aqueous sodium acetate solution. The precipitate was collected by filtration. The product was purified by chromatography on silica gel (hexane/dichloromethane 1:1) to give compound A as a milk white solid (3.127 g, 89.7 \%); mp: $75-77^{\circ} \mathrm{C} ;{ }^{1} \mathrm{H}$ NMR (400 MHz, Acetone- $d_{6}$ ), $\delta(\mathrm{ppm}): 9.56$ (s, 1H), 7.22 (s, 2H), 3.12 (t, $\left.J=6.0 \mathrm{~Hz}, 4 \mathrm{H}\right), 2.74$ (t, $\left.J=6.0 \mathrm{~Hz}, 4 \mathrm{H}\right), 1.93$ (m, 4H); ${ }^{13} \mathrm{C}$ NMR (100 MHz, $\left.\mathrm{CDCl}_{3}\right) \delta$ (ppm): 190.19, 148.00, 129.55, 124.13, 120.43, 50.15, 27.77, 21.38; HRMS (EI+): 201.1153 (calculated: 201.1154). 
4-diphenylamino-benzaldehyde (B). mp: $130-132{ }^{\circ} \mathrm{C} ;{ }^{1} \mathrm{H} \mathrm{NMR}\left(400 \mathrm{MHz}, \mathrm{CDCl}_{3}\right), \delta$ (ppm): 9.81 (s, 1H), 7.68 (d, $J=8.4 \mathrm{~Hz}, 2 \mathrm{H}), 7.34$ (t, $J=7.6 \mathrm{~Hz}, 6 \mathrm{H}), 7.18(\mathrm{~d}, J=6.8 \mathrm{~Hz}, 4 \mathrm{H}), 7.02(\mathrm{~d}, J=8.4 \mathrm{~Hz}, 2 \mathrm{H})$; ${ }^{13} \mathrm{C}$ NMR (100 MHz, $\left.\mathrm{CDCl}_{3}\right) \delta(\mathrm{ppm}):$ 190.55, 153.52, 146.33, 131.45, 129.90, 129.29, 126.47, 125.27, 119.52; HRMS (EI+): 273.1147 (calculated: 273.1154).

II. The synthesis of Diethyl [(5-bromothien-2-yl)methyl]phosphonate (C). A 50 ml flask fitted with a reflux condenser was charged with 2-bromothiophene (6) (4.0 g, $24.2 \mathrm{mmol})$, paraformaldehyde (0.825 g, $27.5 \mathrm{mmol}$ ) and HOAc (12 ml), $\mathrm{HBr}$ in HOAc (5 ml, 40 \%) was added and the solution was heated in a well ventilated fume cupboard at $50^{\circ} \mathrm{C}$ for $3 \mathrm{~h}$. After cooling the solution, the reaction mixture was extracted with $\mathrm{CH}_{2} \mathrm{Cl}_{2}(100 \mathrm{ml})$ and washed with cold water $(50 \mathrm{ml})$, dried over $\mathrm{Na}_{2} \mathrm{SO}_{4}$, and the solvent was finally evaporated to afford a brown oil 7. Then, the brown oil was mixed with triethylphosphite (5.06 g, $30.5 \mathrm{mmol}$ ). The viscous solution was stirred at $150{ }^{\circ} \mathrm{C}$ overnight. Evaporation of the excess of triethylphosphite, the dark oil $\mathbf{C}$ was got. This was used without further purification in the next step.

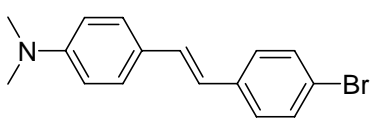

$1 \mathbf{a}$

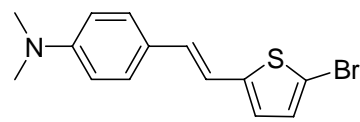

$2 \mathbf{a}$

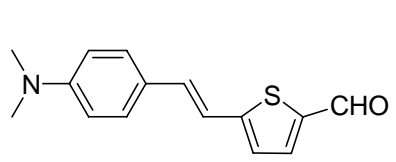

3a

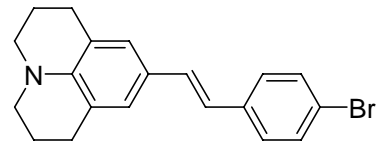

1b

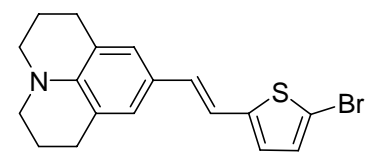

2b

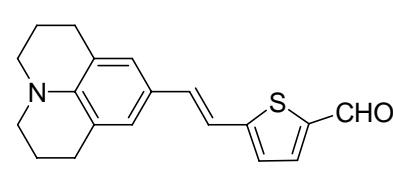

3b

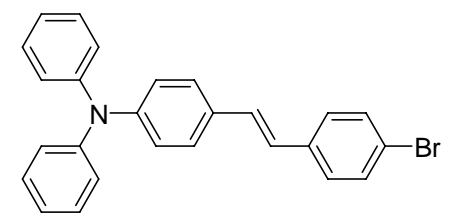

1c

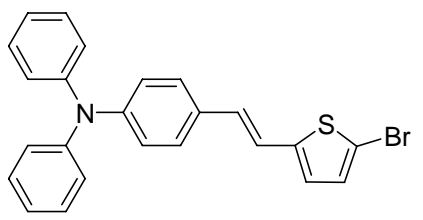

2c

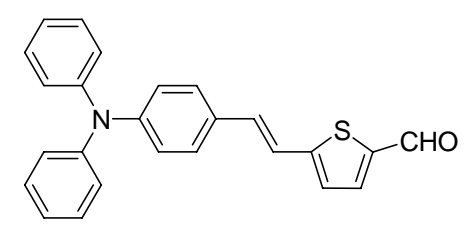

3c

Chart 1. Structures of D- $\pi$-A molecules 1-3 


\section{General procedure for the synthesis of $\{4-[2-(4-b r o m o-p h e n y l)-v i n y l]-p h e n y l\}$-dimethyl-amine}

(1a). A mixture of 4-bromobenzylbromide (2.237 g, $8.95 \mathrm{mmol})$ and triethylphosphite (1.783 g, 10.74

mmol) under $\mathrm{N}_{2}$ was heated at $150{ }^{\circ} \mathrm{C}$ for $3 \mathrm{~h}$. The solution was cooled and then anhydrous DMF (10 ml) was added. The solution was placed in an ice bath for $15 \mathrm{~min}$ and then $\mathrm{NaH}(0.620 \mathrm{~g},(>52 \%$ in mineral oil), $13.42 \mathrm{mmol}$ ) was added. The mixture was stirred for $20 \mathrm{~min}$ and then 4-N,N-dimethylamino-benzaldehyde (1.0 g, $6.71 \mathrm{mmol})$ in anhydrous DMF (8 ml) was slowly added. The ice bath was removed and the solution was stirred for $12 \mathrm{~h}$ before it was decanted into ice water. The precipitate was collected by filtration. Further purification was performed by recrystallization from $\mathrm{CHCl}_{3} / \mathrm{CH}_{3} \mathrm{OH}$ 1:1 (v/v) to afford compound $1 \mathrm{a}(1.535 \mathrm{~g}, 75.5 \%$ ) as a milk white glistening sheet solid; mp: $245-246{ }^{\circ} \mathrm{C} ;{ }^{1} \mathrm{H}$ NMR (400 MHz, DMSO- $\left.d_{6}\right), \delta$ (ppm): 7.51 (d, $\left.J=8.8 \mathrm{~Hz}, 2 \mathrm{H}\right), 7.48(\mathrm{~d}, J=9.6 \mathrm{~Hz}$, 2H), 7.43 (d, $J=8.8 \mathrm{~Hz}, 2 \mathrm{H}), 7.17$ (d, $J=16.4 \mathrm{~Hz}, 1 \mathrm{H}), 6.95$ (d, $J=16.8 \mathrm{~Hz}, 1 \mathrm{H}), 6.72(\mathrm{~d}, J=8.8 \mathrm{~Hz}$, 2H), 2.93 (s, 6H); ${ }^{13} \mathrm{C}$ NMR (100 MHz, $\left.\mathrm{CDCl}_{3}\right), \delta$ (ppm): 150.42, 137.35, 131.80, 129.72, 127.85, 127.65, 125.50, 123.16, 120.29, 112.59, 40.62; HRMS (EI+): 301.0466 (calculated: 301.0466).

\section{9-[2-(4-bromo-phenyl)-vinyl]-2,3,6,7-tetrahydro-1H,5H-Pyrido[3,2,1-ij]quinoline (1b). mp:}

$125-127{ }^{\circ} \mathrm{C} ;{ }^{1} \mathrm{H}$ NMR (400 MHz, Acetone- $\left.d_{6}\right), \delta(\mathrm{ppm}): 7.47$ (d, $\left.J=8.8 \mathrm{~Hz}, 2 \mathrm{H}\right), 7.45$ (d, $J=8.8 \mathrm{~Hz}$, 2H), 7.06 (d, $J=16.4 \mathrm{~Hz}, 1 \mathrm{H}), 6.97$ (s, 2H), 6.87 (d, $J=16.0 \mathrm{~Hz}, 1 \mathrm{H}), 3.18$ (t, $J=5.6 \mathrm{~Hz}, 4 \mathrm{H}), 2.72$ (t, $J$ $=6.4 \mathrm{~Hz}, 4 \mathrm{H}), 1.93(\mathrm{~m}, 4 \mathrm{H}) ;{ }^{13} \mathrm{C}$ NMR (100 MHz, $\left.\mathrm{CDCl}_{3}\right), \delta(\mathrm{ppm}):$ 143.07, 137.57, 131.72, 130.03, 127.49, 125.67, 124.48, 122.19, 121.53, 119.93, 50.13, 27.88, 22.13; HRMS (EI+): 353.0771 (calculated: 353.0779).

\{4-[2-(4-bromo-phenyl)-vinyl]-phenyl\}-diphenyl-amine (1c). mp: $193-194{ }^{\circ} \mathrm{C} ;{ }^{1} \mathrm{H} \mathrm{NMR}(400 \mathrm{MHz}$, $\left.\mathrm{CDCl}_{3}\right), \delta(\mathrm{ppm}): 7.46(\mathrm{~d}, J=8.4 \mathrm{~Hz}, 2 \mathrm{H}), 7.36(\mathrm{t}, J=8.4 \mathrm{~Hz}, 4 \mathrm{H}), 7.29-7.25(\mathrm{~m}, 4 \mathrm{H}), 7.11(\mathrm{~d}, J=7.6$ $\mathrm{Hz}, 4 \mathrm{H}), 7.04$ (t, $J=8.0 \mathrm{~Hz}, 5 \mathrm{H}), 6.91$ (d, $J=16.4 \mathrm{~Hz}, 1 \mathrm{H}) ;{ }^{13} \mathrm{C}$ NMR (100 MHz, $\left.\mathrm{CDCl}_{3}\right), \delta(\mathrm{ppm})$ : 
147.79, 147.63, 136.76, 131.90, 131.14, 129.48, 129.06, 127.91, 127.59, 125.80, 124.76, 123.56, 123.32,

121.03; HRMS (EI+): 425.0778 (calculated: 425.0779).

\section{General procedure for the synthesis of $\{4-[2-(5$-bromo-thiophene)-vinyl]-phenyl\}-dimeth-}

ylamine (2a). A suspension of $\mathrm{NaH}$ (232 mg, (> 52 \% in mineral oil), $5.03 \mathrm{mmol}$ ) was thoroughly washed with anhydrous hexane and then suspended in anhydrous THF (8 ml). A solution of diethyl 5-bromothien-2-ylmethanephosphonate (1.053 g, $3.36 \mathrm{mmol}$ ) in THF (5 ml) was added under nitrogen to this suspension, followed after $10 \mathrm{~min}$ by the addition of a solution of $4-N, N$-dimethylaminobenzaldehyde (500 mg, $3.36 \mathrm{mmol}$ ) in the same solvent ( $8 \mathrm{ml}$ ). The mixture was cautiously heated on an oil-bath at $50{ }^{\circ} \mathrm{C}$ until the evolution of hydrogen had ceased, and then at reflux for $3 \mathrm{~h}$. The reaction mixture was then cooled, poured into ice water $(100 \mathrm{ml})$ and the aqueous phase was extracted with ether $(4 \times 50 \mathrm{ml})$. The organic phases were combined, washed with water and dried over $\mathrm{Na}_{2} \mathrm{SO}_{4}$, and the solvent was finally evaporated to afford a dark solid. Further purification was performed by recrystallization from $\mathrm{CHCl}_{3} / \mathrm{CH}_{3} \mathrm{OH}$ 1:1 (v/v) to afford compound $\mathbf{2 a}(797 \mathrm{mg}, 77.3 \%$ ) as a yellow glistening sheet solid; mp: $158-160{ }^{\circ} \mathrm{C} ;{ }^{1} \mathrm{H}$ NMR (400 MHz, $\left.\mathrm{CDCl}_{3}\right), \delta$ (ppm): 7.35 (d, $\left.J=8.8 \mathrm{~Hz}, 2 \mathrm{H}\right)$, 6.94-6.90 (m, 2H), 6.76 (d, $J=16.0 \mathrm{~Hz} 1 \mathrm{H}), 6.72-6.70$ (m, 3H), 2.30 (s, 6H); ${ }^{13} \mathrm{C}$ NMR (100 MHz, $\left.\mathrm{CDCl}_{3}\right), \delta$ (ppm): 150.38, 145.82, 130.50, 129.36, 127.67, 125.02, 124.76, 117.15, 112.55, 109.61, 40.57; HRMS (EI+): 307.0025 (calculated: 307.0030).

\{4-[2-(5-bromo-thiophene)-vinyl]-phenyl\}-diphenyl-amine (2c). mp: $138-140{ }^{\circ} \mathrm{C} ;{ }^{1} \mathrm{H}$ NMR $(400$ $\left.\mathrm{MHz}, \mathrm{CDCl}_{3}\right), \delta$ (ppm): 7.32-7.24 (m, 6H), 7.10 (d, $\left.J=8.0 \mathrm{~Hz}, 4 \mathrm{H}\right), 7.06-7.00$ (m, 4H), 6.96-6.93 (t, 2H), 6.77-6.73 (m, 2H); ${ }^{13} \mathrm{C}$ NMR (100 MHz, $\left.\mathrm{CDCl}_{3}\right), \delta$ (ppm): 147.80, 147.61, 145.12, 130.72, 130.62, 129.49, 128.56, 127.39, 125.77, 124.80, 123.51, 123.37, 119.64, 110.65; HRMS (EI+): 431.0333 (calculated: 431.0343). 


\section{General procedure for the synthesis of 5-[2-(4-dimethylamino-phenyl)-vinyl]-thiophene-2-}

carbaldehyde (3a). A solution of $n$-BuLi $2.57 \mathrm{M}$ in hexane $(2.7 \mathrm{ml}, 7.0 \mathrm{mmol})$ was added slowly to a solution of compound $\mathbf{2 a}(307 \mathrm{mg}, 1.0 \mathrm{mmol})$ in anhydrous THF $(15 \mathrm{ml})$ at $0{ }^{\circ} \mathrm{C}$ under $\mathrm{N}_{2}$ atmosphere. The mixture was stirred for $0.5 \mathrm{~h}$ at $0{ }^{\circ} \mathrm{C}$ before dropwise addition of anhydrous DMF (0.38 ml, 5.0 $\mathrm{mmol}$ ), and the reaction was stirred for $3 \mathrm{~h}$ at room temperature, and then the mixture was poured into a saturated aqueous solution of ammonium chloride $(100 \mathrm{ml})$. The solution was extracted with acetic ether $(3 \times 50 \mathrm{ml})$, washed with water $(3 \times 50 \mathrm{ml})$, dried over $\mathrm{Na}_{2} \mathrm{SO}_{4}$ and evaporated in vacuo. The residue was purified by column chromatography on silica gel (hexane/dichloromethane/ethyl acetate 6:3:0.4) to afford a red solid 3a (157 mg, $61.2 \%$ ); mp: $156-158{ }^{\circ} \mathrm{C} ;{ }^{1} \mathrm{H}$ NMR (400 MHz, Acetone- $\left.d_{6}\right), \delta(\mathrm{ppm}): 9.86$ (s, $\left.1 \mathrm{H}\right)$, $7.84(\mathrm{~d}, J=4.0 \mathrm{~Hz}, 1 \mathrm{H}), 7.49(\mathrm{~d}, J=8.8 \mathrm{~Hz}, 2 \mathrm{H}), 7.24$ (d, $J=4.0 \mathrm{~Hz}, 1 \mathrm{H}), 7.21(\mathrm{~d}, J=2.4 \mathrm{~Hz}, 2 \mathrm{H}), 6.76$ (d, $J=8.8 \mathrm{~Hz}, 2 \mathrm{H}), 3.01$ (s, 6H); ${ }^{13} \mathrm{C}$ NMR (100 MHz, $\left.\mathrm{CDCl}_{3}\right), \delta(\mathrm{ppm}): 182.49,154.38,150.94,140.34$, 137.74, 133.73, 128.50, 125.17, 124.04, 116.32, 112.33, 40.41; HRMS (EI+): 257.0871 (calculated: 257.0874).

5-\{9-(2,3,6,7-tetrahydro-1H,5H-Pyrido[3,2,1-ij]quinoline)-vinyl\}-thiophene-2-carbaldehyde (3b). mp: $142-144{ }^{\circ} \mathrm{C} ;{ }^{1} \mathrm{H}$ NMR (400 MHz, $\mathrm{CDCl}_{3}$ ), $\delta$ (ppm): 9.84 (s, $\left.1 \mathrm{H}\right), 7.83$ (d, $\left.J=4.0 \mathrm{~Hz}, 1 \mathrm{H}\right), 7.20$ (d, $J$ $=3.6 \mathrm{~Hz}, 1 \mathrm{H}), 7.13(\mathrm{~d}, J=16.0 \mathrm{~Hz}, 1 \mathrm{H}), 7.07(\mathrm{~d}, J=16.0 \mathrm{~Hz}, 1 \mathrm{H}), 7.01(\mathrm{~s}, 2 \mathrm{H}), 3.22(\mathrm{t}, J=6.0 \mathrm{~Hz}, 4 \mathrm{H})$, $2.73(\mathrm{t}, J=6.0 \mathrm{~Hz}, 4 \mathrm{H}), 1.93(\mathrm{~m}, 4 \mathrm{H}) ;{ }^{13} \mathrm{C} \mathrm{NMR}$ (100 MHz, $\left.\mathrm{CDCl}_{3}\right), \delta(\mathrm{ppm}): 182.39,154.90,143.88$, 139.94, 137.85, 134.18, 126.30, 124.73, 123.00, 121.42, 115.31, 50.08, 27.86, 21.92; HRMS (EI+): 309.1187 (calculated: 309.1187).

5-[2-(4-diphenylamino-phenyl)-vinyl]-thiophene-2-carbaldehyde (3c). mp: $141-143{ }^{\circ} \mathrm{C} ;{ }^{1} \mathrm{H}$ NMR (400 MHz, $\left.\mathrm{CDCl}_{3}\right), \delta$ (ppm): $9.77(\mathrm{~s}, 1 \mathrm{H}), 7.58$ (d, $\left.J=4.0 \mathrm{~Hz}, 1 \mathrm{H}\right), 7.29$ (d, $\left.J=8.8 \mathrm{~Hz}, 2 \mathrm{H}\right), 7.21$ (m, 4H), 7.06-7.00 (m, 9H), 6.96 (d, $J=8.8 \mathrm{~Hz}, 2 \mathrm{H}) ;{ }^{13} \mathrm{C}$ NMR (100 MHz, $\left.\mathrm{CDCl}_{3}\right), \delta(\mathrm{ppm}): 182.61,153.34$, 
148.69, 147.35, 141.16, 137.52, 132.79, 129.58, 128.07, 126.07, 125.14, 123.76, 122.82, 118.91; HRMS (EI+): 381.1187 (calculated: 381.1187).

2. ${ }^{1} \mathrm{H}$ NMR and ${ }^{13} \mathrm{C}$ NMR spectra of compounds 1-3 and intermediates A, B. 

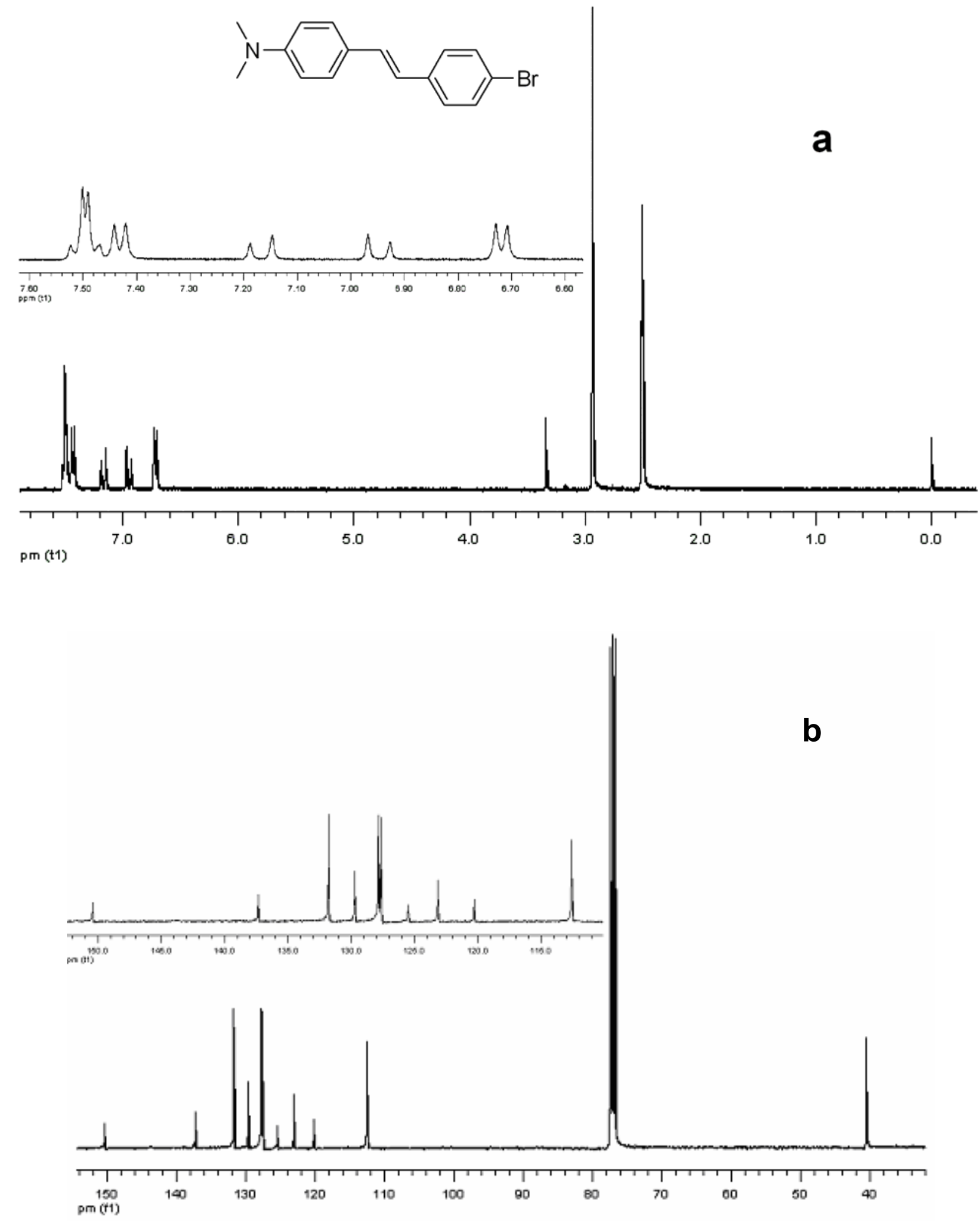

Figure S1. ${ }^{1} \mathrm{H}$ NMR (a) and ${ }^{13} \mathrm{C}$ NMR (b) spectra of compound $\mathbf{1 a}$. 

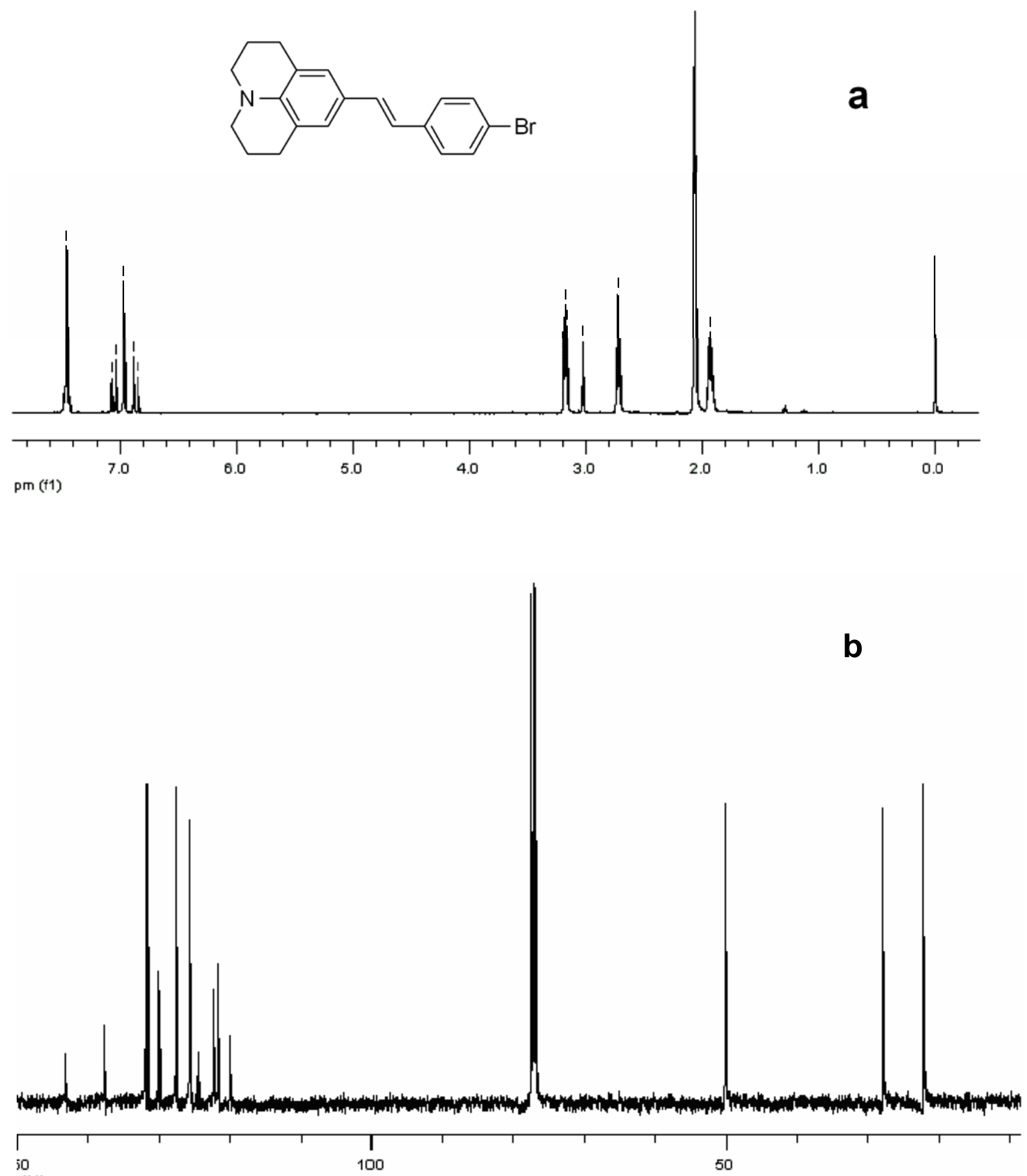

(f1)

Figure S2. ${ }^{1} \mathrm{H}$ NMR (a) and ${ }^{13} \mathrm{C}$ NMR (b) spectra of compound $\mathbf{1 b}$. 

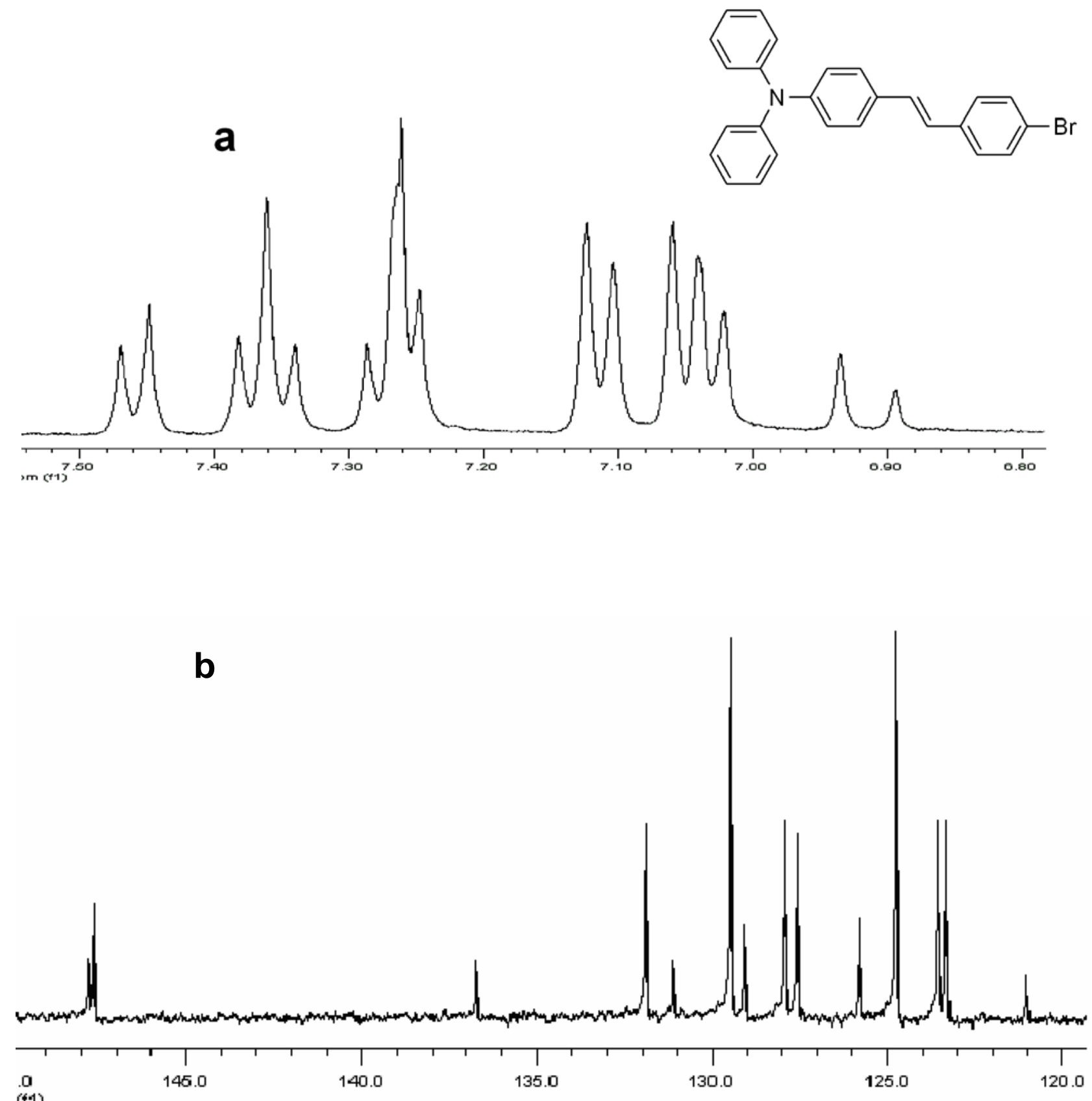

Figure S3. ${ }^{1} \mathrm{H}$ NMR (a) and ${ }^{13} \mathrm{C}$ NMR (b) spectra of compound $\mathbf{1 c}$. 


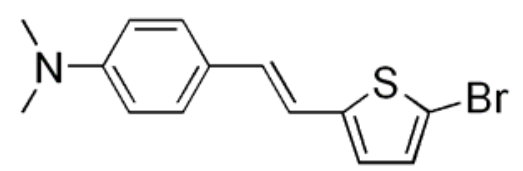

a
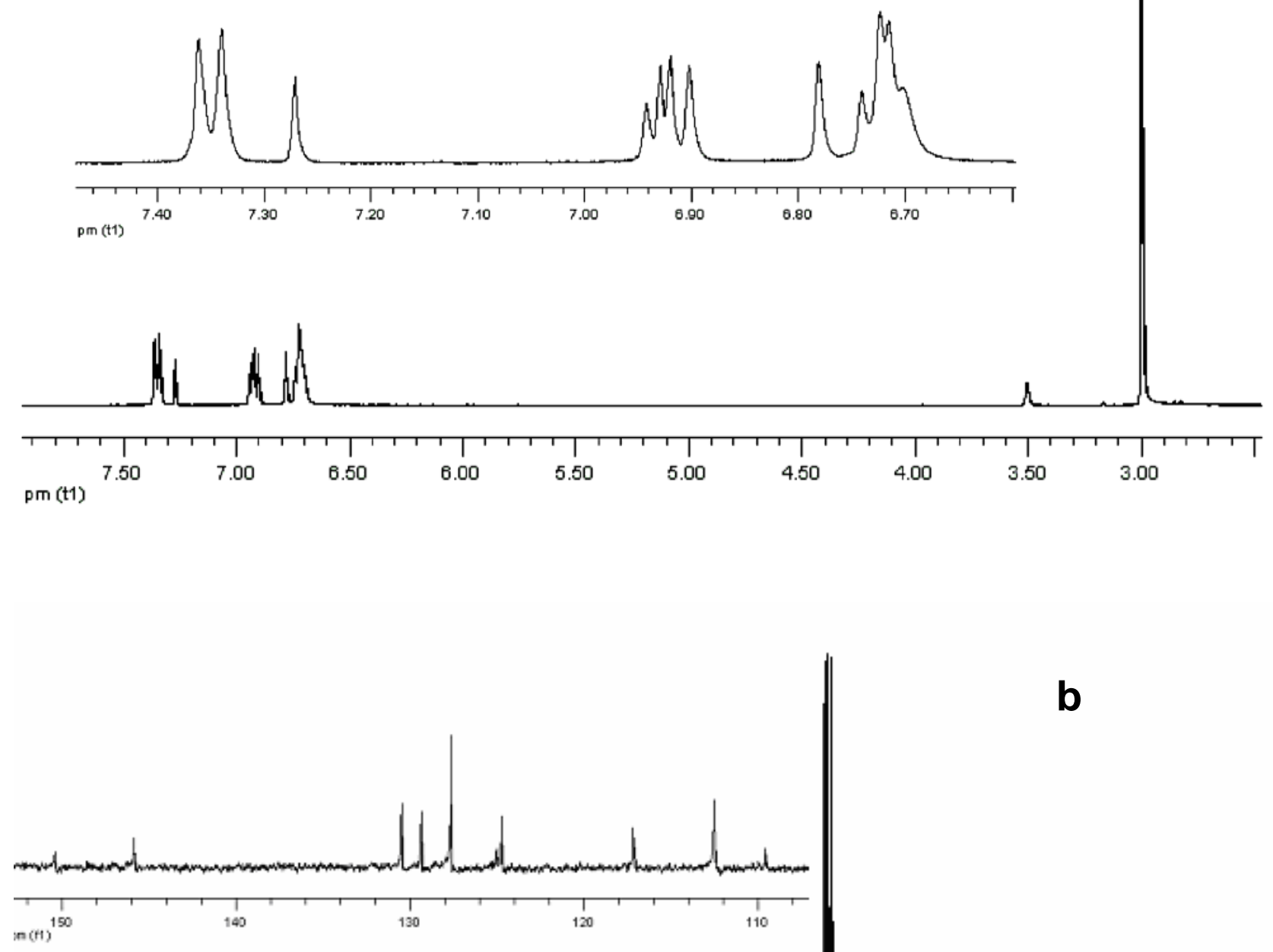

b

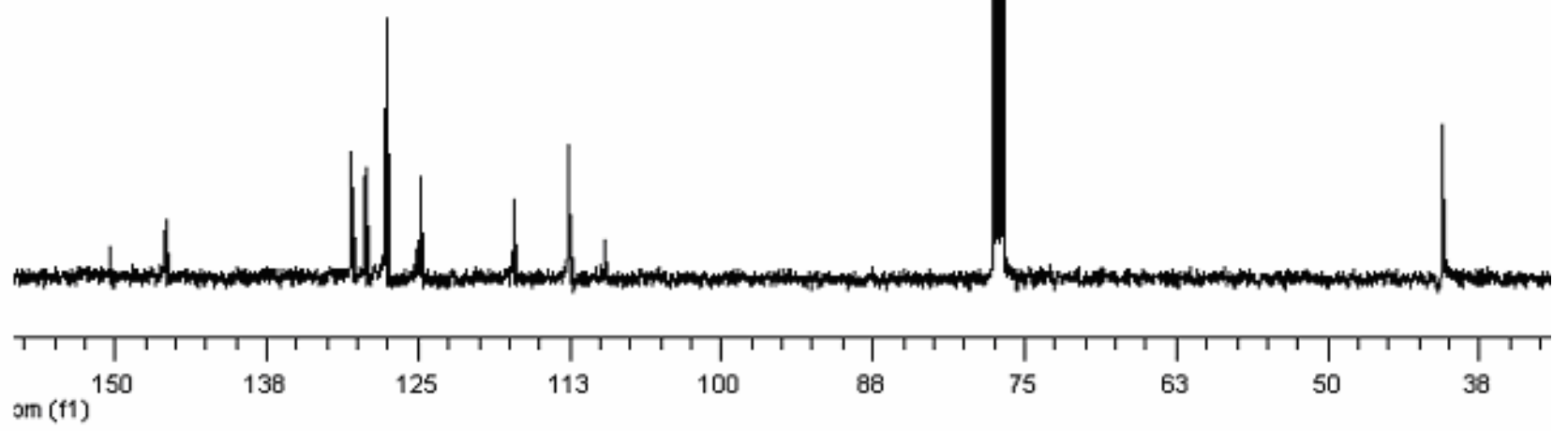

Figure S4. ${ }^{1} \mathrm{H}$ NMR (a) and ${ }^{13} \mathrm{C}$ NMR (b) spectra of compound $\mathbf{2 a}$. 


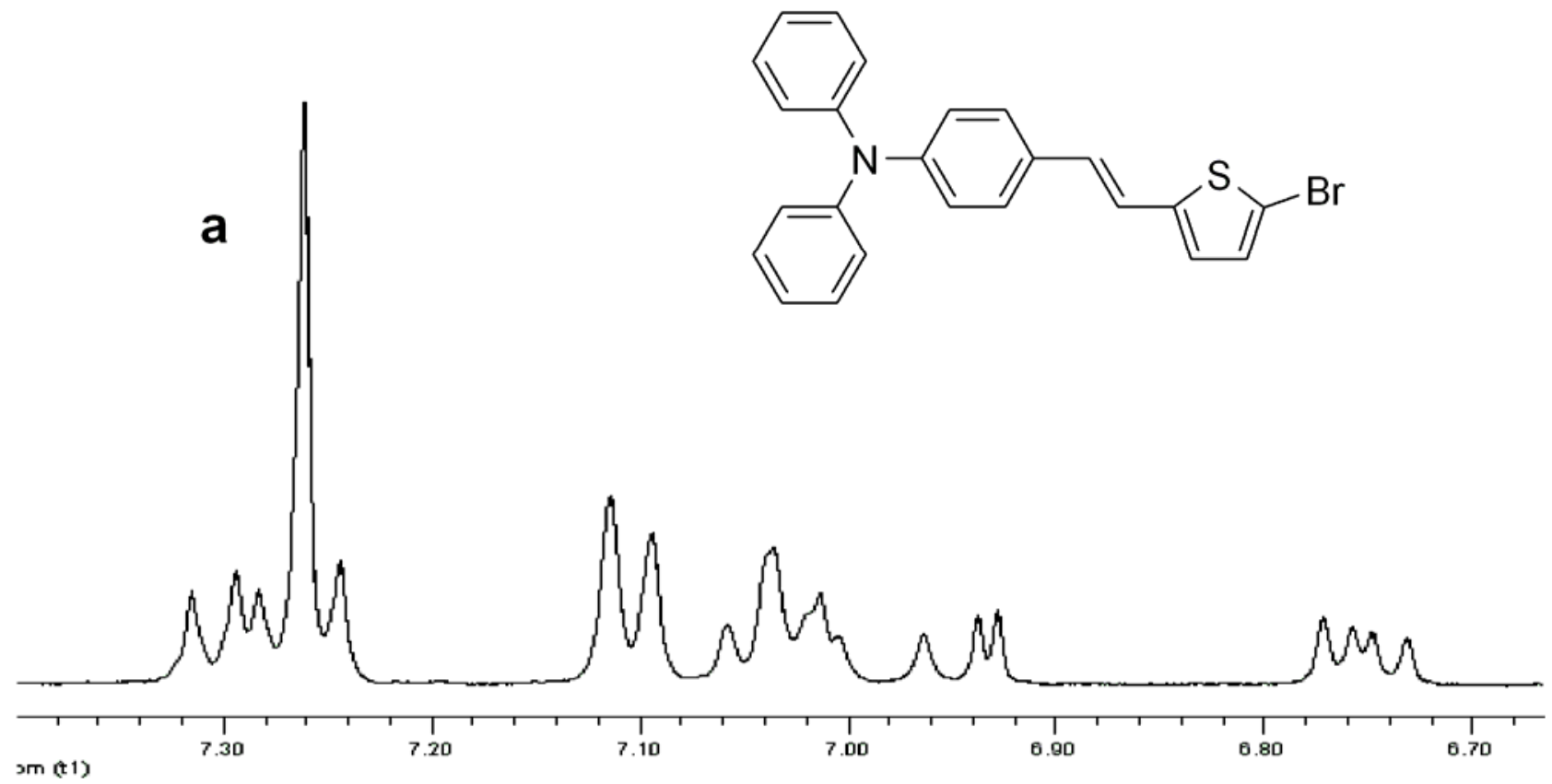

b

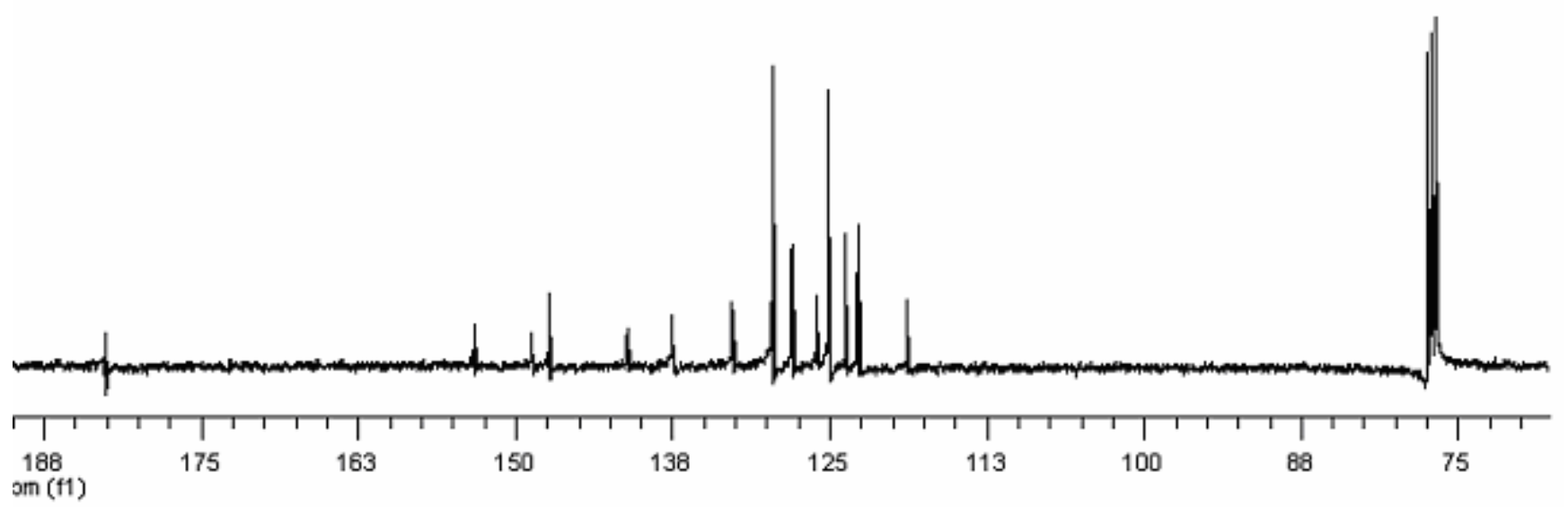

Figure S5. ${ }^{1} \mathrm{H}$ NMR (a) and ${ }^{13} \mathrm{C}$ NMR (b) spectra of compound $2 \mathbf{c}$. 

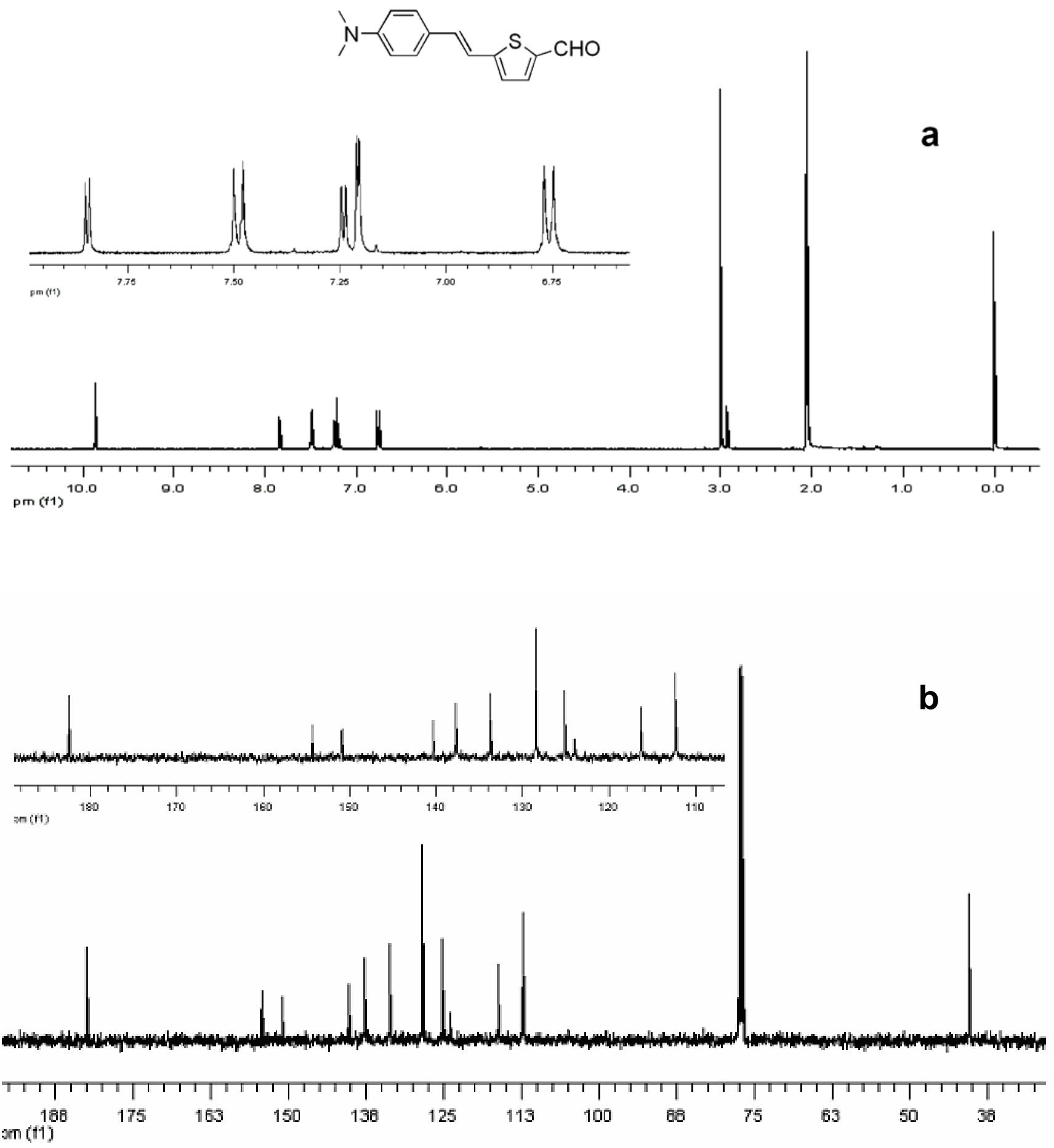

Figure S6. ${ }^{1} \mathrm{H}$ NMR (a) and ${ }^{13} \mathrm{C}$ NMR (b) spectra of compound $\mathbf{3 a}$. 

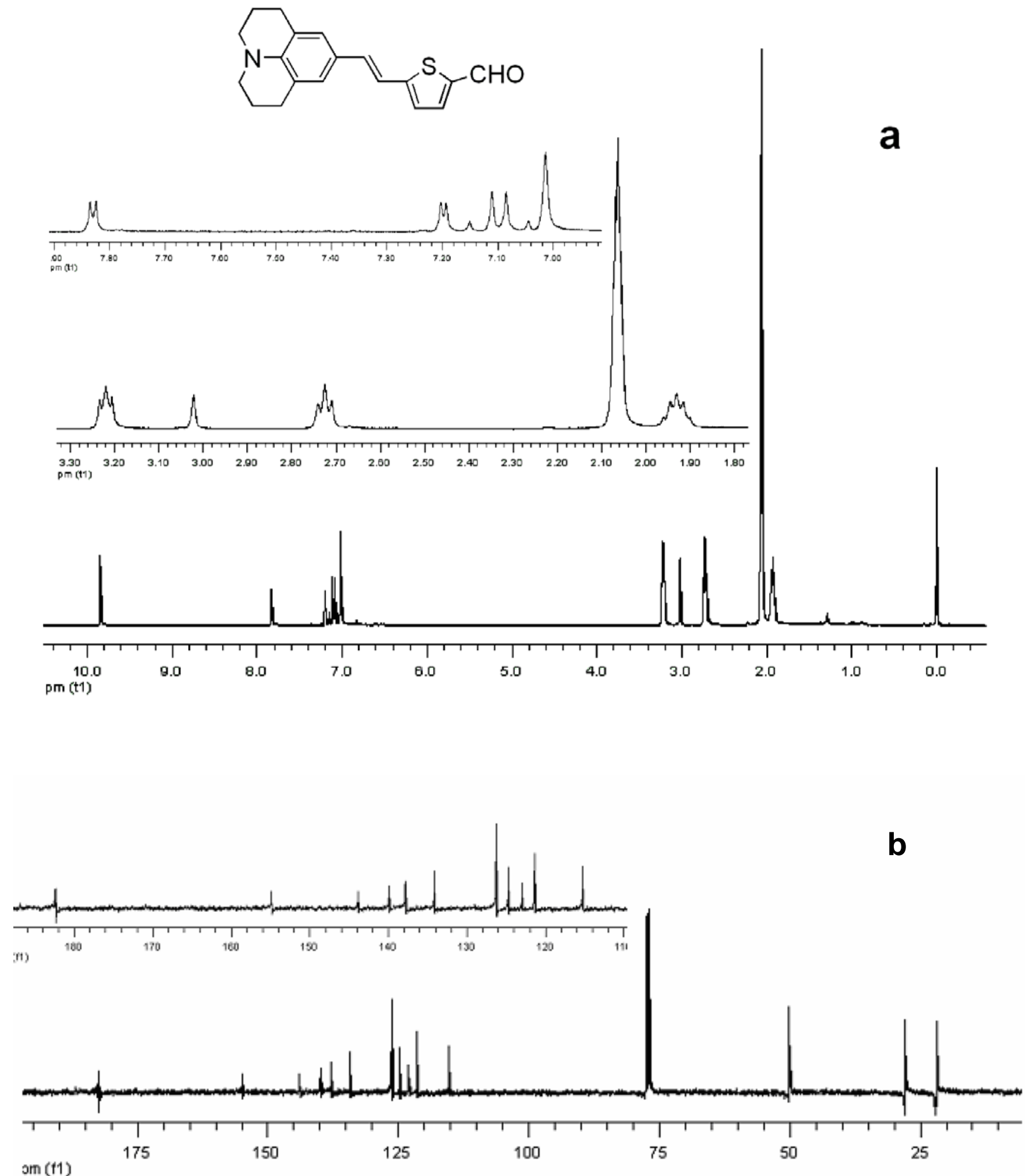

Figure S7. ${ }^{1} \mathrm{H}$ NMR (a) and ${ }^{13} \mathrm{C}$ NMR (b) spectra of compound $\mathbf{3 b}$. 

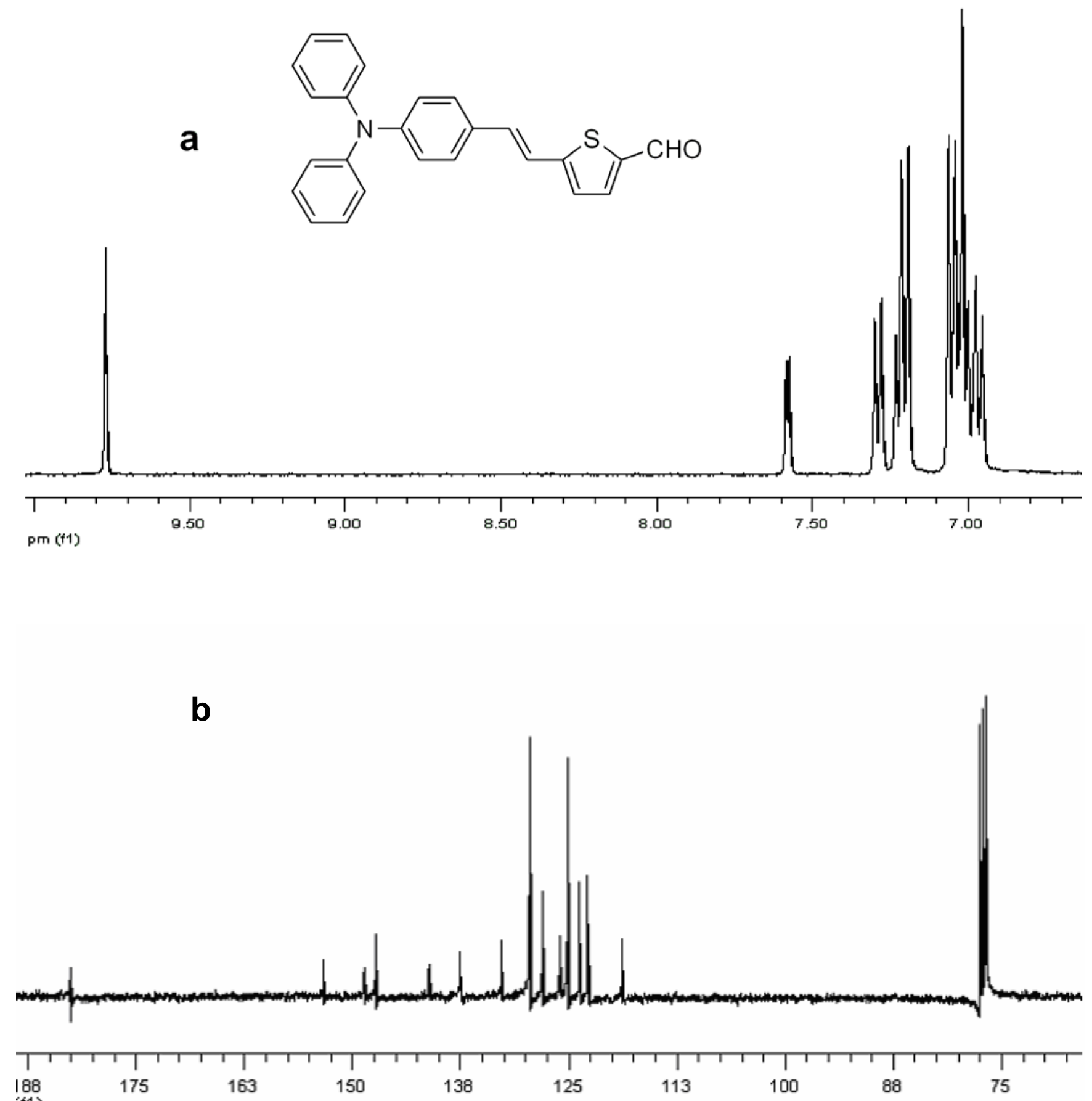

(f1)

Figure S8. ${ }^{1} \mathrm{H}$ NMR (a) and ${ }^{13} \mathrm{C}$ NMR (b) spectra of compound 3c. 


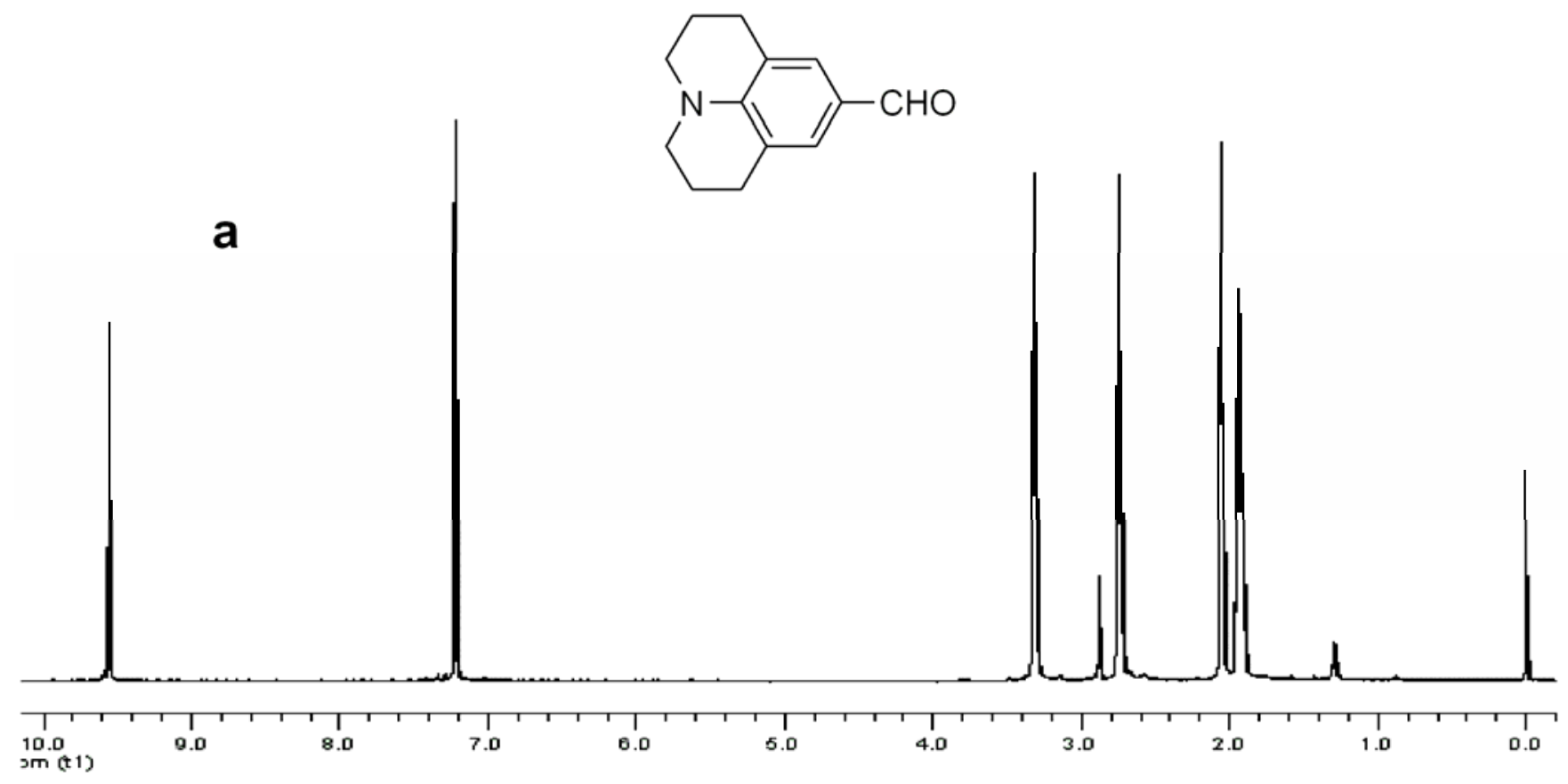

b

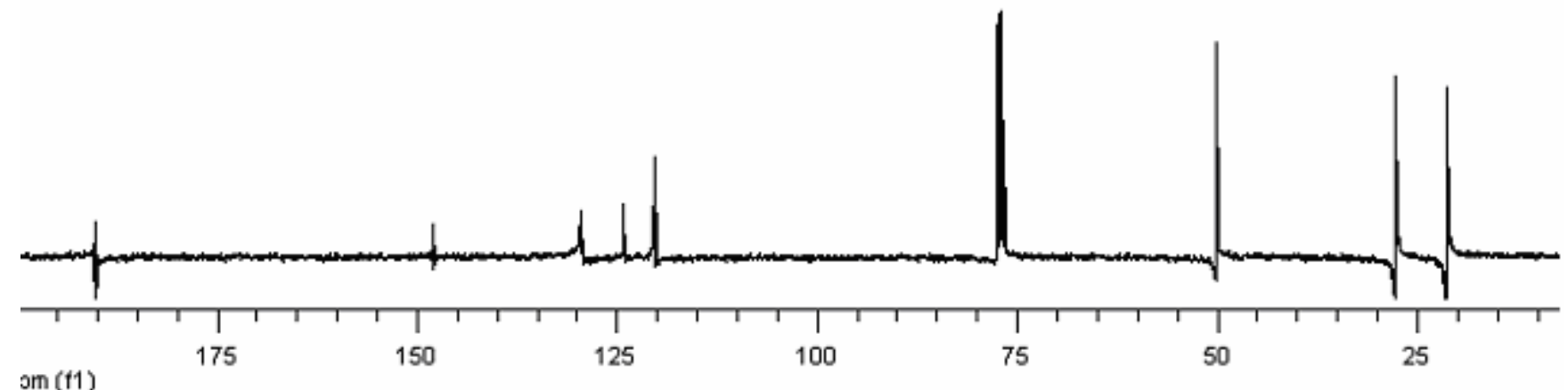

Figure S9. ${ }^{1} \mathrm{H}$ NMR (a) and ${ }^{13} \mathrm{C}$ NMR (b) spectra of compound $\mathbf{A}$. 

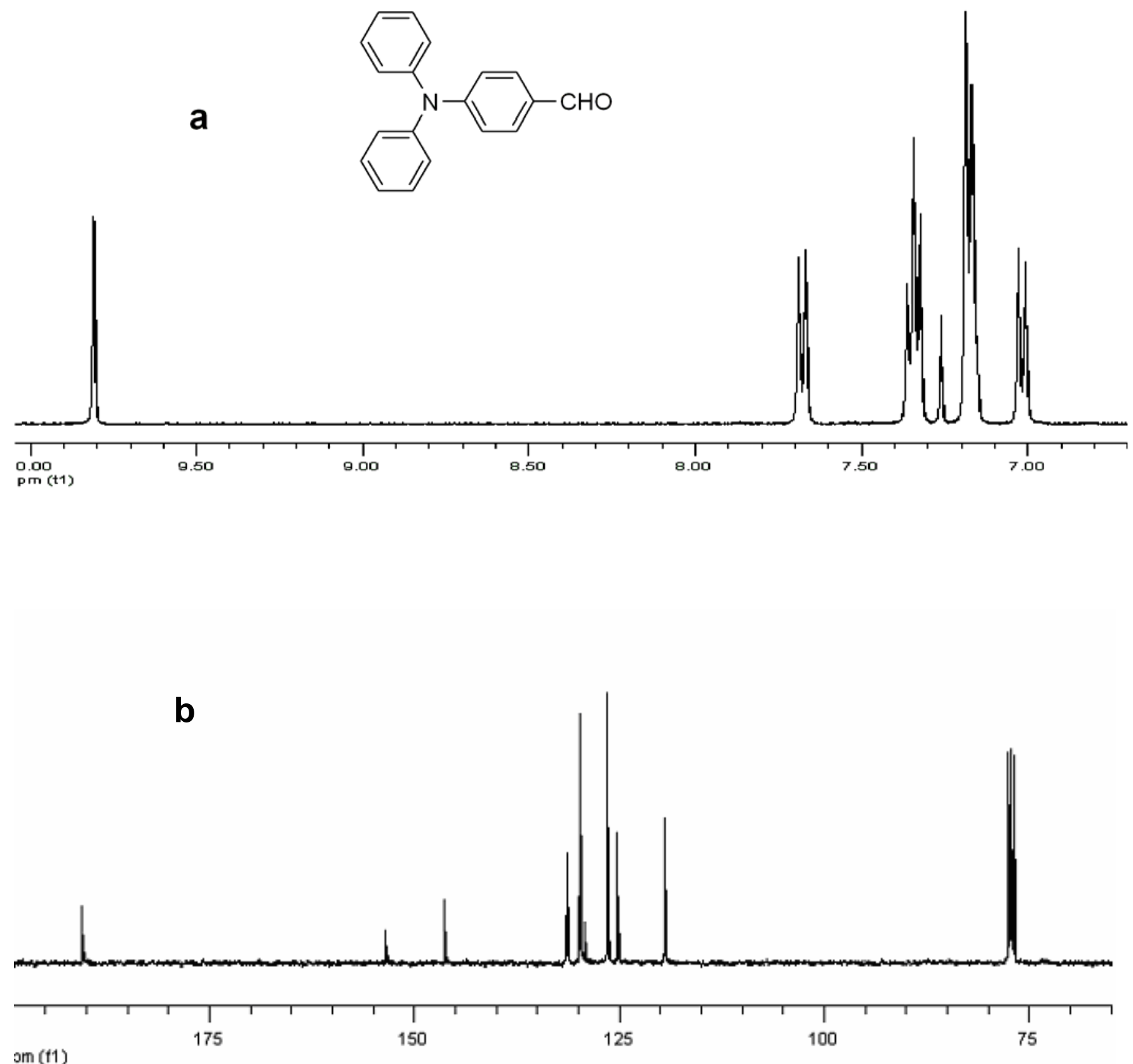

Figure S10. ${ }^{1} \mathrm{H}$ NMR (a) and ${ }^{13} \mathrm{C}$ NMR (b) spectra of compound $\mathbf{B}$.

\section{Electrochemistry of compounds 1-3.}




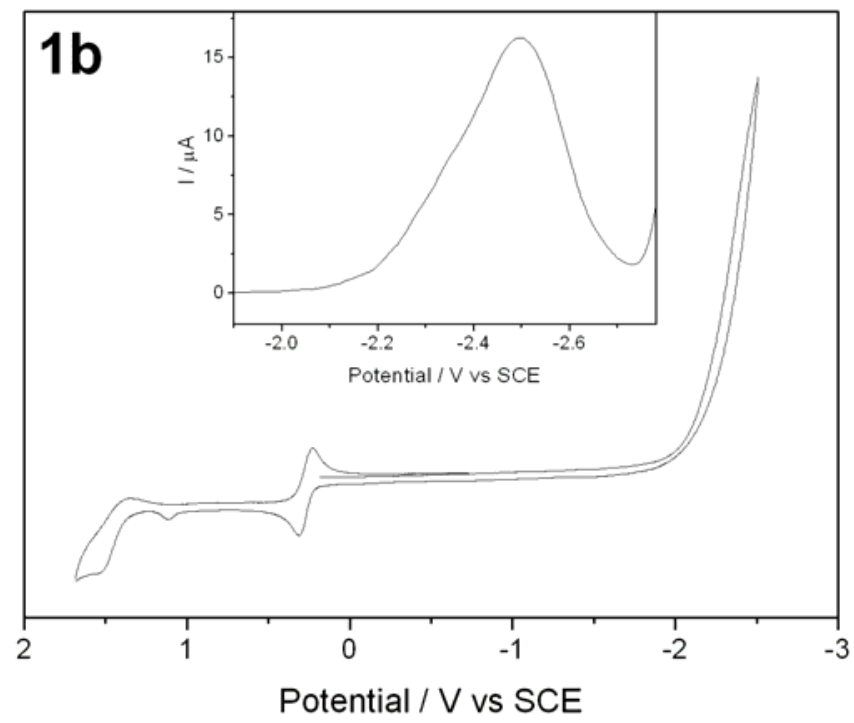

Figure S11. Cyclic voltammogram of $\mathbf{1 b}$ with sample concentration of $1 \times 10^{-3} \mathrm{M}$ in a $1: 1 \mathrm{PhH} / \mathrm{MeCN}$ solution using a platinum working electrode and $\mathrm{Ag} / \mathrm{Ag}^{+}$reference electrode, scan rate $100 \mathrm{mV} \mathrm{s}^{-1}$. Inset: DPV of $\mathbf{1 b}$ in 1:1 $\mathrm{PhH} / \mathrm{MeCN}$ using a platinum working electrode and $\mathrm{Ag} / \mathrm{Ag}^{+}$reference electrode.

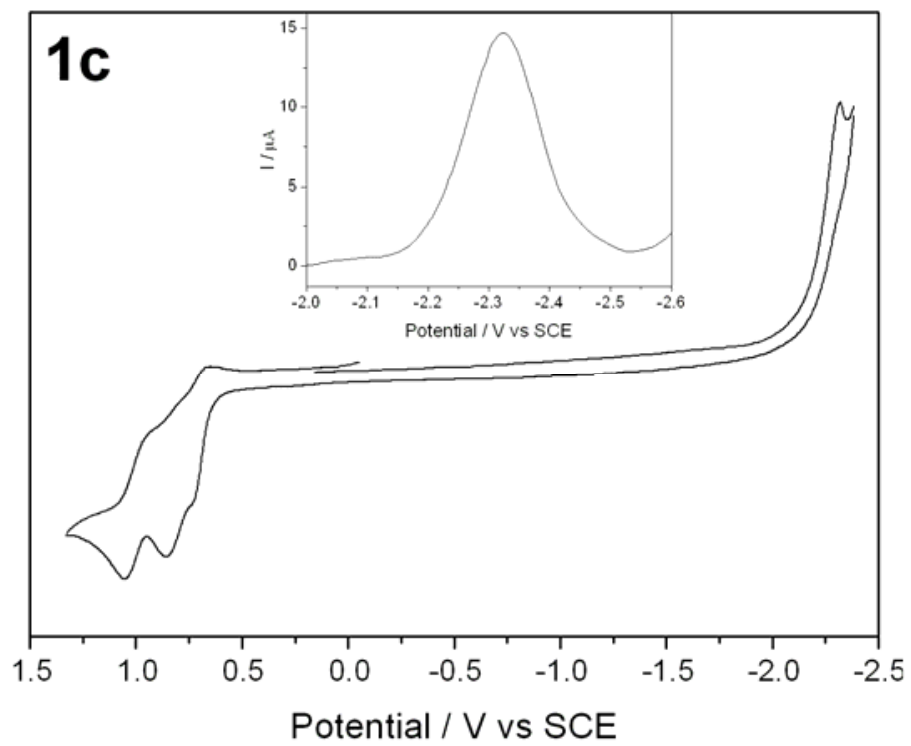

Figure S12. Cyclic voltammogram of $1 \mathrm{c}$ with sample concentration of $1 \times 10^{-3} \mathrm{M}$ in $1: 1 \mathrm{PhH} / \mathrm{MeCN}$ using a platinum working electrode and $\mathrm{Ag} / \mathrm{Ag}^{+}$reference electrode, scan rate $100 \mathrm{mV} \mathrm{s}^{-1}$. Inset: DPV of $1 \mathrm{c}$ in 1:1 $\mathrm{PhH} / \mathrm{MeCN}$ using a platinum working electrode and $\mathrm{Ag} / \mathrm{Ag}^{+}$reference electrode. 


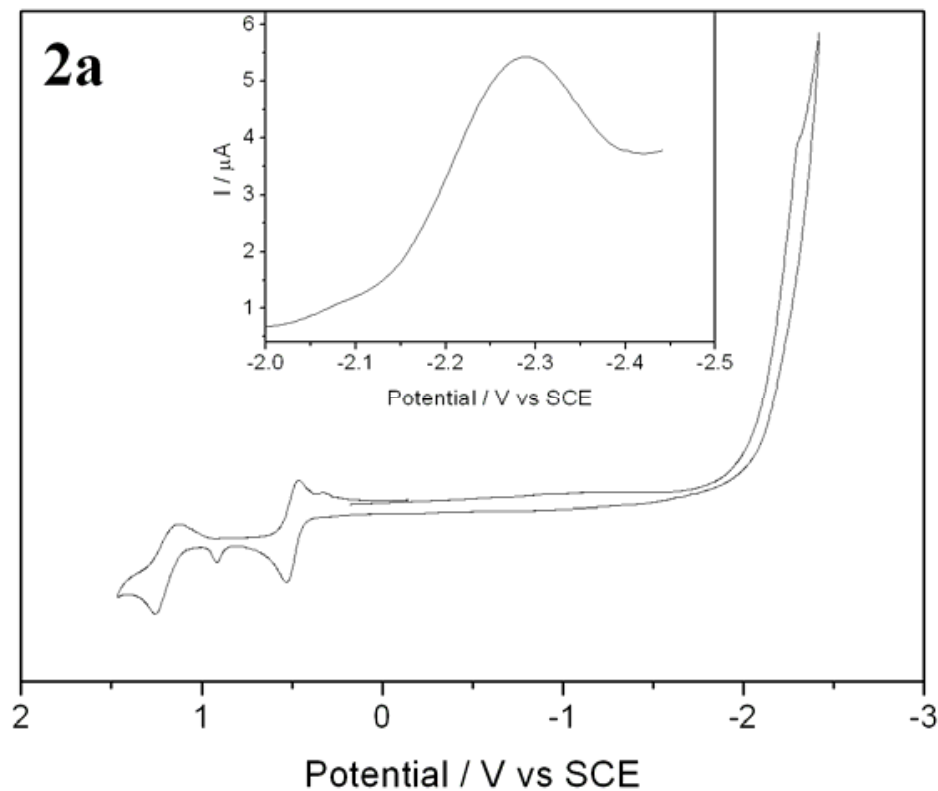

Figure S13. Cyclic voltammogram of 2 a with sample concentration of $1 \times 10^{-3} \mathrm{M}$ in $1: 1 \mathrm{PhH} / \mathrm{MeCN}$ using a platinum working electrode and $\mathrm{Ag} / \mathrm{Ag}^{+}$reference electrode, scan rate $100 \mathrm{mV} \mathrm{s}^{-1}$. Inset: DPV of $\mathbf{2 a}$ in 1:1 $\mathrm{PhH} / \mathrm{MeCN}$ using a platinum working electrode and $\mathrm{Ag} / \mathrm{Ag}^{+}$reference electrode.

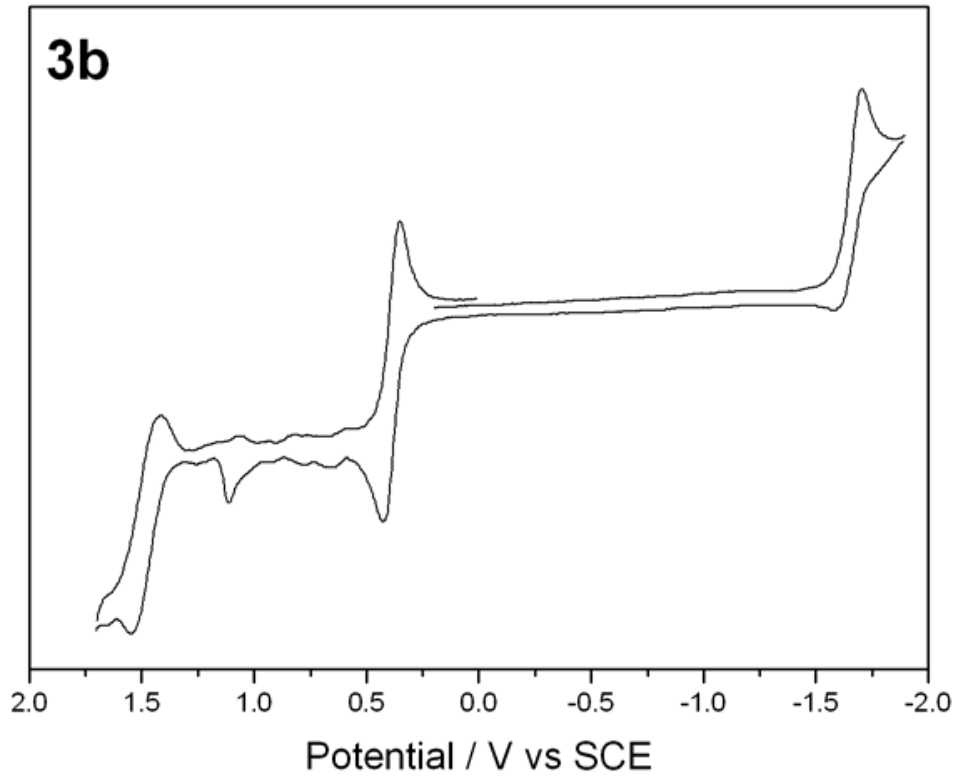

Figure S14. Cyclic voltammogram of $\mathbf{3 b}$ with sample concentration of $1 \times 10^{-3} \mathrm{M}$ in 1:1 $\mathrm{PhH} / \mathrm{MeCN}$ using a platinum working electrode and $\mathrm{Ag} / \mathrm{Ag}^{+}$reference electrode, scan rate $100 \mathrm{mV} \mathrm{s}^{-1}$. 


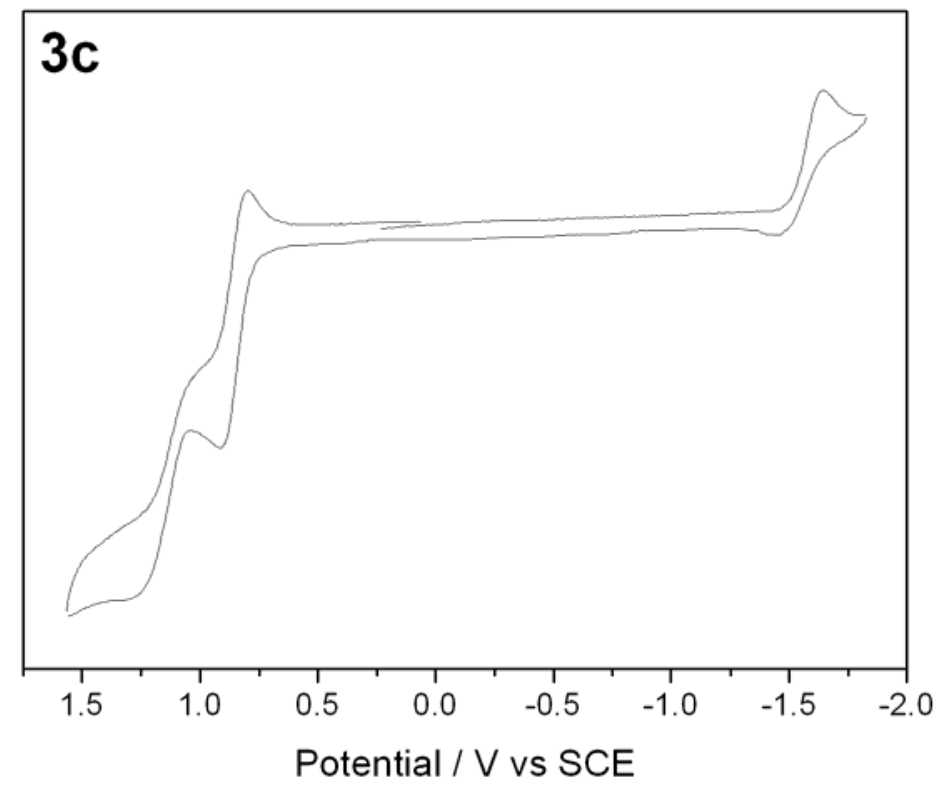

Figure S15. Cyclic voltammogram of $3 \mathbf{c}$ with sample concentration of $1 \times 10^{-3} \mathrm{M}$ in $1: 1 \mathrm{PhH} / \mathrm{MeCN}$ using a platinum working electrode and $\mathrm{Ag} / \mathrm{Ag}^{+}$reference electrode, scan rate $100 \mathrm{mV} \mathrm{s}^{-1}$. 
4. Electrogenerated chemiluminescence of compounds 1-3.

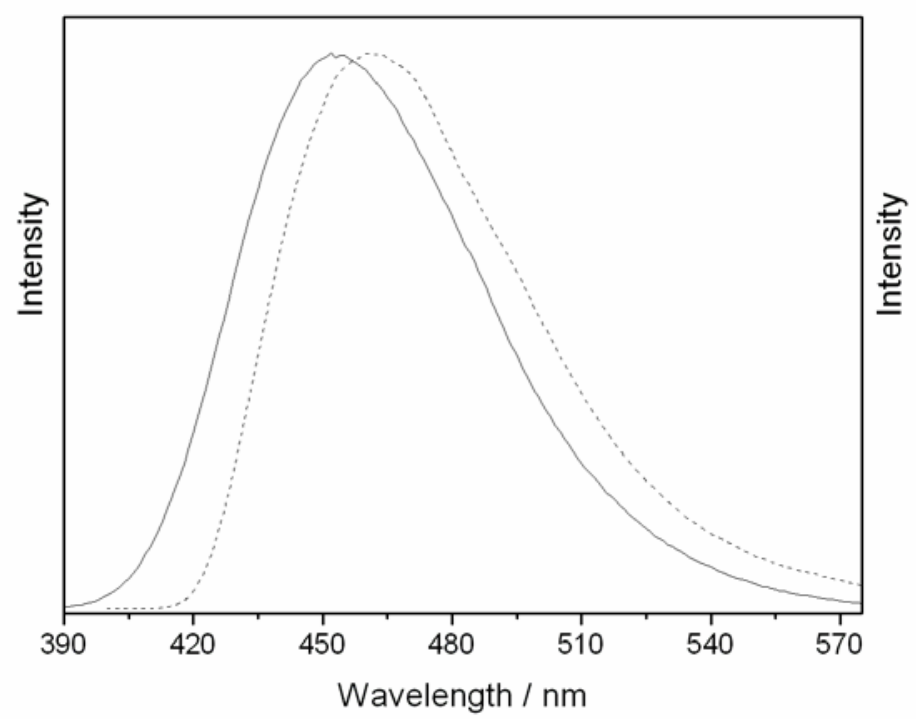

Figure S16. ECL $\left(10^{-3} \mathrm{M}\right.$, dash) and fluorescence $\left(10^{-5} \mathrm{M}\right.$, solid) spectra of 1a in 1:1 $\mathrm{PhH} / \mathrm{MeCN}$.

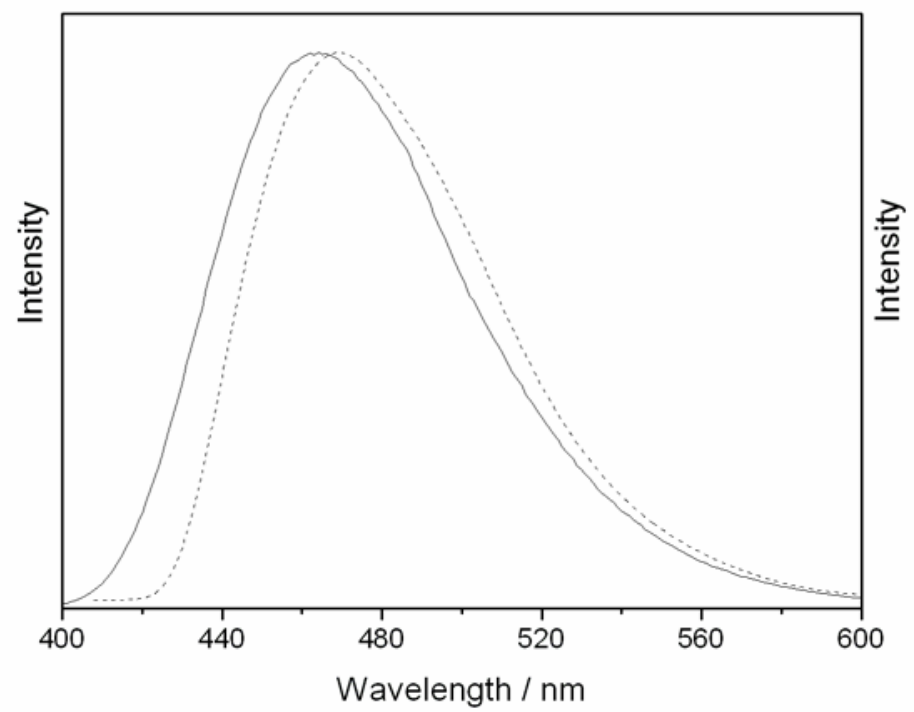

Figure S17. ECL $\left(10^{-3} \mathrm{M}\right.$, dash) and fluorescence $\left(10^{-5} \mathrm{M}\right.$, solid) spectra of $1 \mathrm{c}$ in $1: 1 \mathrm{PhH} / \mathrm{MeCN}$. 


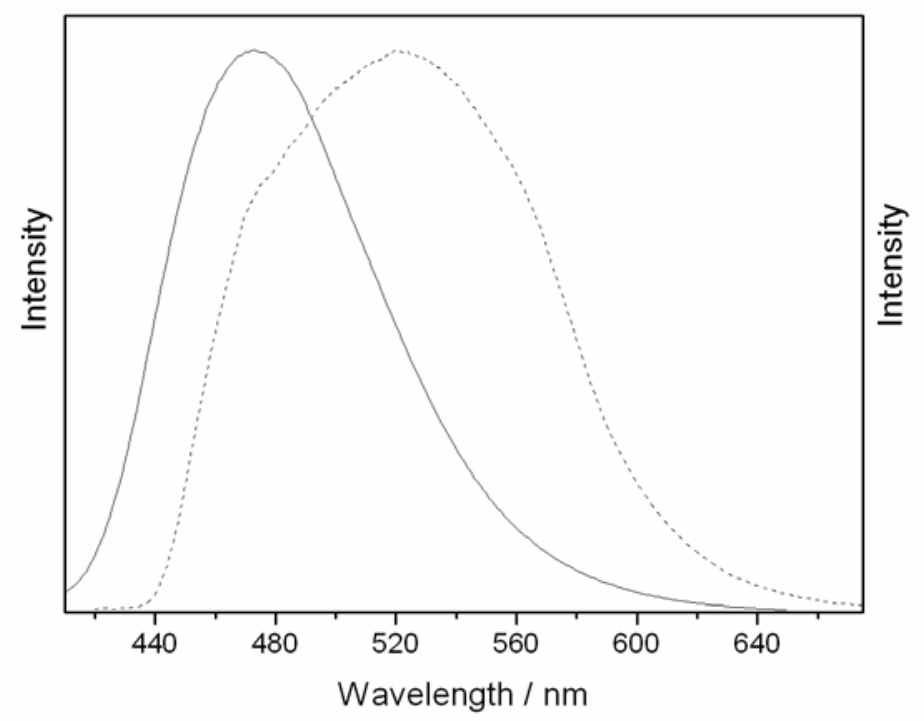

Figure S18. ECL $\left(10^{-3} \mathrm{M}\right.$, dash) and fluorescence $\left(10^{-5} \mathrm{M}\right.$, solid) spectra of $2 \mathbf{c}$ in $1: 1 \mathrm{PhH} / \mathrm{MeCN}$.

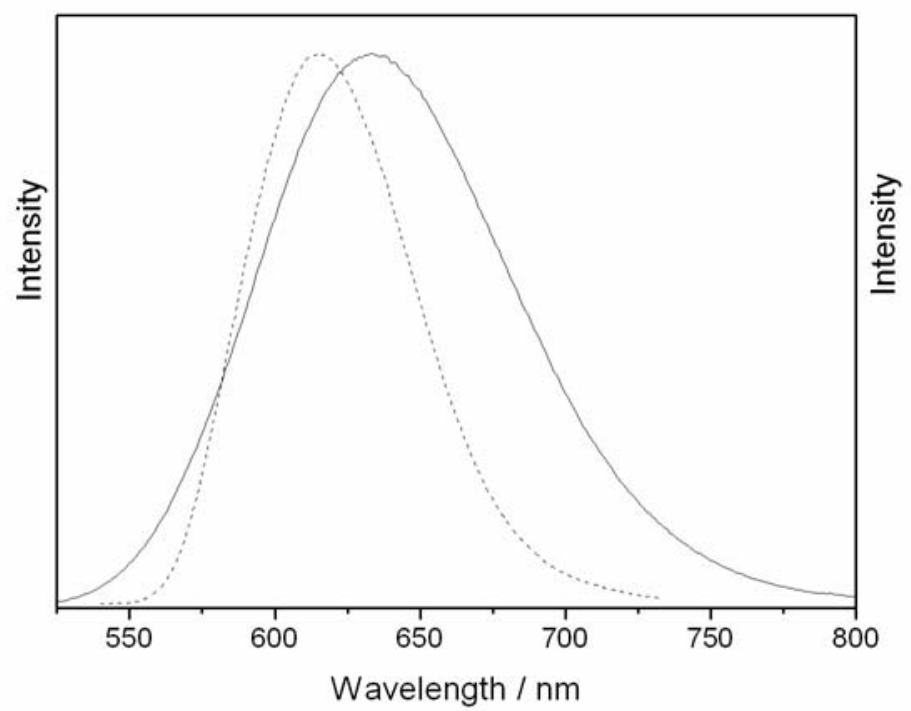

Figure S19. ECL $\left(10^{-3} \mathrm{M}\right.$, dash) and fluorescence $\left(10^{-5} \mathrm{M}\right.$, solid) spectra of $\mathbf{3 b}$ in $1: 1 \mathrm{PhH} / \mathrm{MeCN}$. 


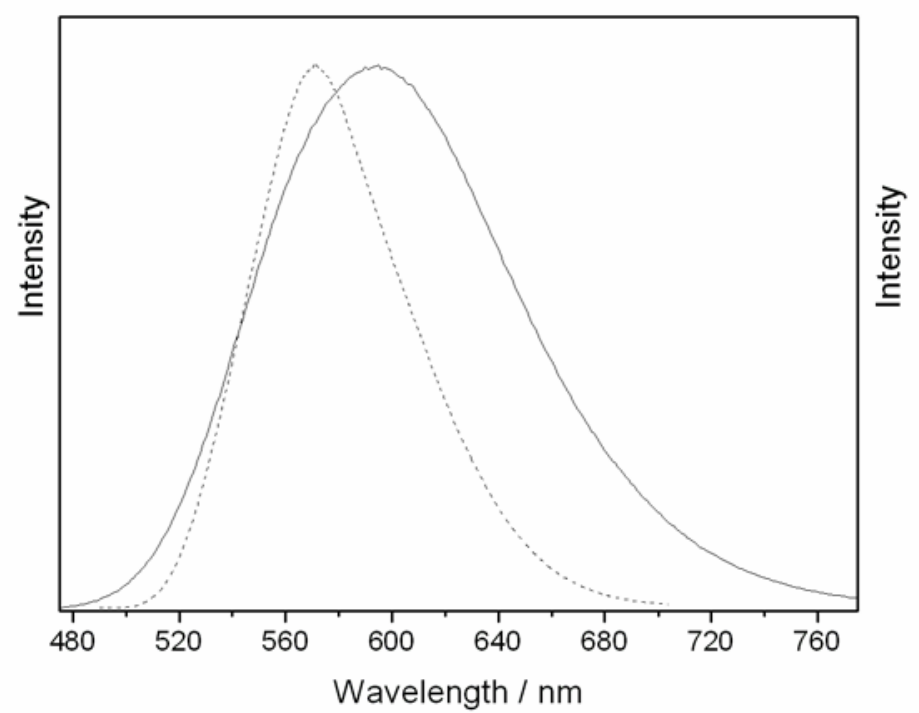

Figure S20. ECL $\left(10^{-3} \mathrm{M}\right.$, dash) and fluorescence $\left(10^{-5} \mathrm{M}\right.$, solid) spectra of $\mathbf{3 c}$ in 1:1 $\mathrm{PhH} / \mathrm{MeCN}$. 


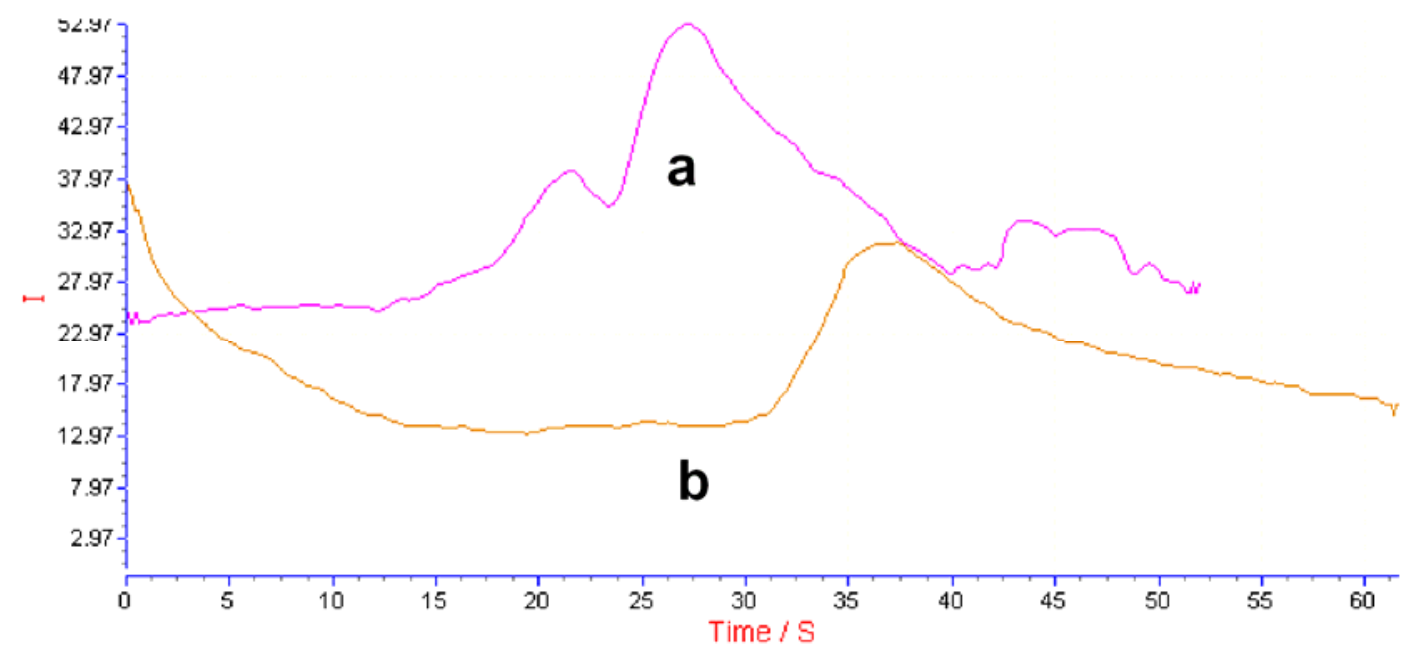

Figure S21. ECL emission spectra of $1 \times 10^{-3} \mathrm{M}$ (a) and $1 \times 10^{-5} \mathrm{M}$ (b) $\mathbf{1 b}$ solutions in 1:1 $\mathrm{PhH} / \mathrm{MeCN}$ containing $0.05 \mathrm{M}$ $\mathrm{TBAPF}_{6}$ as an electrolyte. The potential was stepped from $-2.7 \mathrm{~V}$ to $+1.5 \mathrm{~V}$.

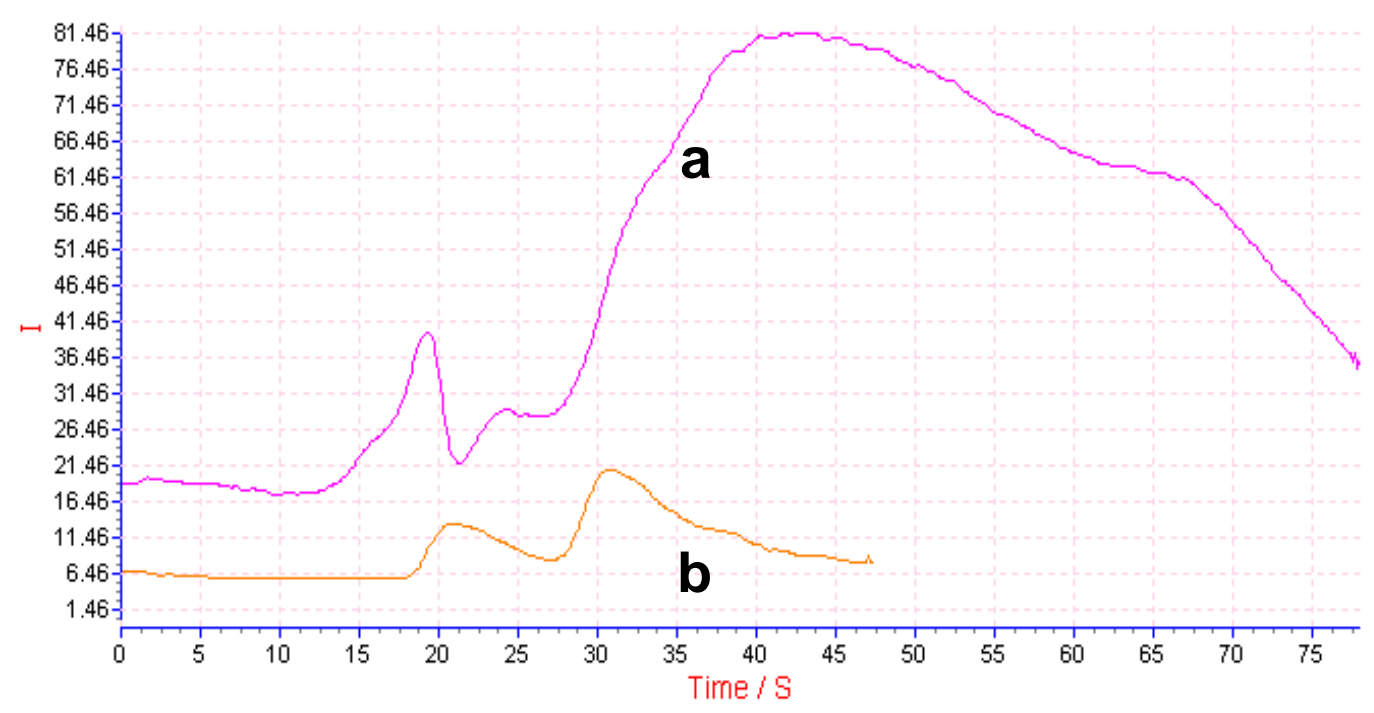

Figure S22. ECL emission spectra of $1 \times 10^{-3} \mathrm{M}$ (a) and $1 \times 10^{-5} \mathrm{M}$ (b) 2a solutions in $1: 1 \mathrm{PhH} / \mathrm{MeCN}$ containing $0.05 \mathrm{M}$ $\mathrm{TBAPF}_{6}$ as an electrolyte. The potential was stepped from $-2.7 \mathrm{~V}$ to $+1.5 \mathrm{~V}$. 


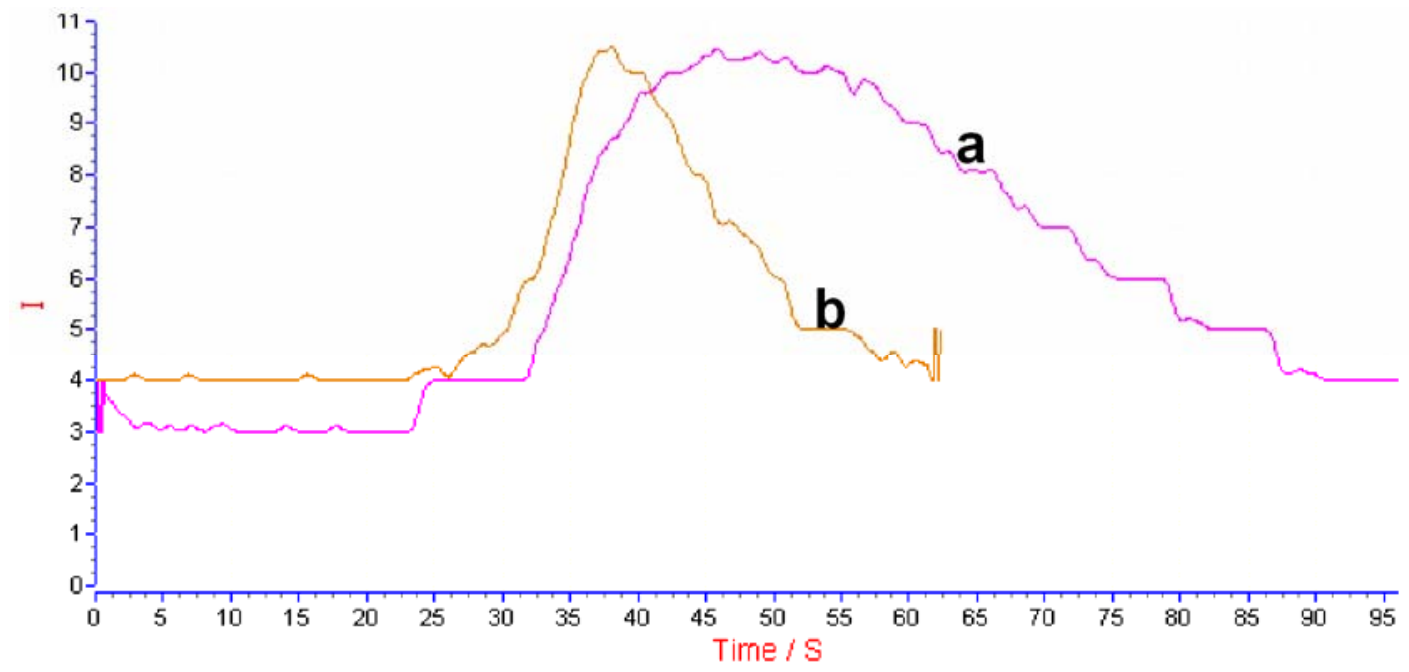

Figure S23. ECL emission spectra of $1 \times 10^{-3} \mathrm{M}$ (a) and $1 \times 10^{-5} \mathrm{M}$ (b) 3c solutions in 1:1 $\mathrm{PhH} / \mathrm{MeCN}$ containing $0.05 \mathrm{M}$ $\mathrm{TBAPF}_{6}$ as an electrolyte. The potential was stepped from $-2.7 \mathrm{~V}$ to $+1.5 \mathrm{~V}$. 
5. Fluorescence and absorption spectra of compound 3a with different concentrations in 1:1

\section{PhH/MeCN.}

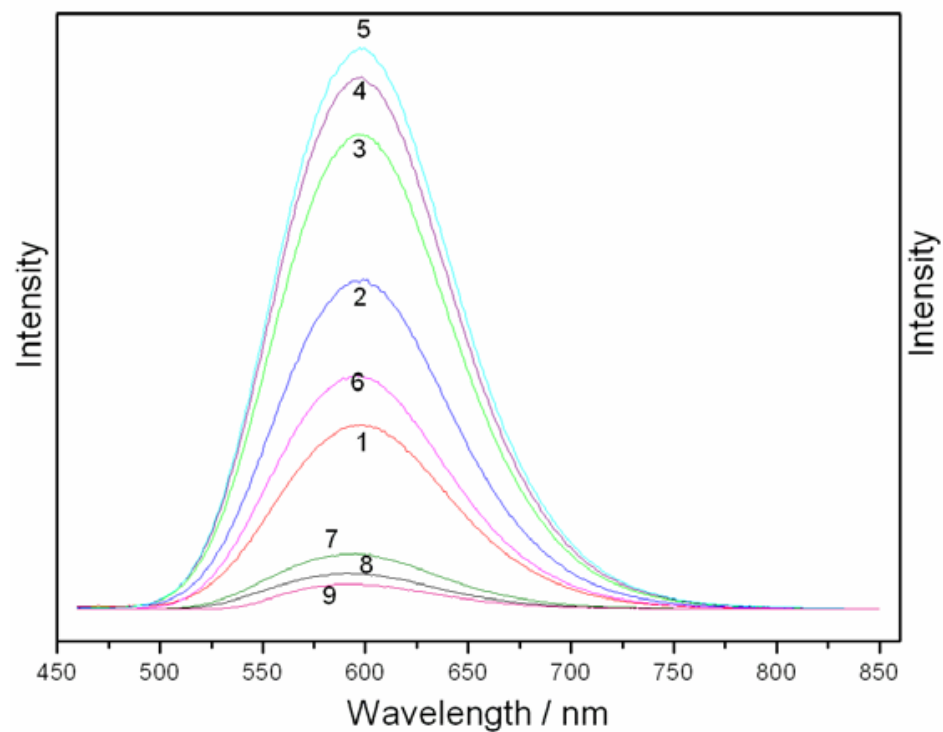

Figure S24. Fluorescence spectra of compound 3a (1) $1 \times 10^{-5} \mathrm{M}$ (red), (2) $2.5 \times 10^{-5} \mathrm{M}$ (blue), (3) $5 \times 10^{-5} \mathrm{M}$ (green), (4) $7.5 \times 10^{-5} \mathrm{M}$ (purple), (5) $1 \times 10^{-4} \mathrm{M}$ (cyan), (6) $2.5 \times 10^{-4} \mathrm{M}$ (magenta), (7) $5 \times 10^{-4} \mathrm{M}$ (olive), (8) $7.5 \times 10^{-4} \mathrm{M}$ (black), (9) $7.5 \times 10^{-3} \mathrm{M}$ (pink) in 1:1 $\mathrm{PhH} / \mathrm{MeCN}$.

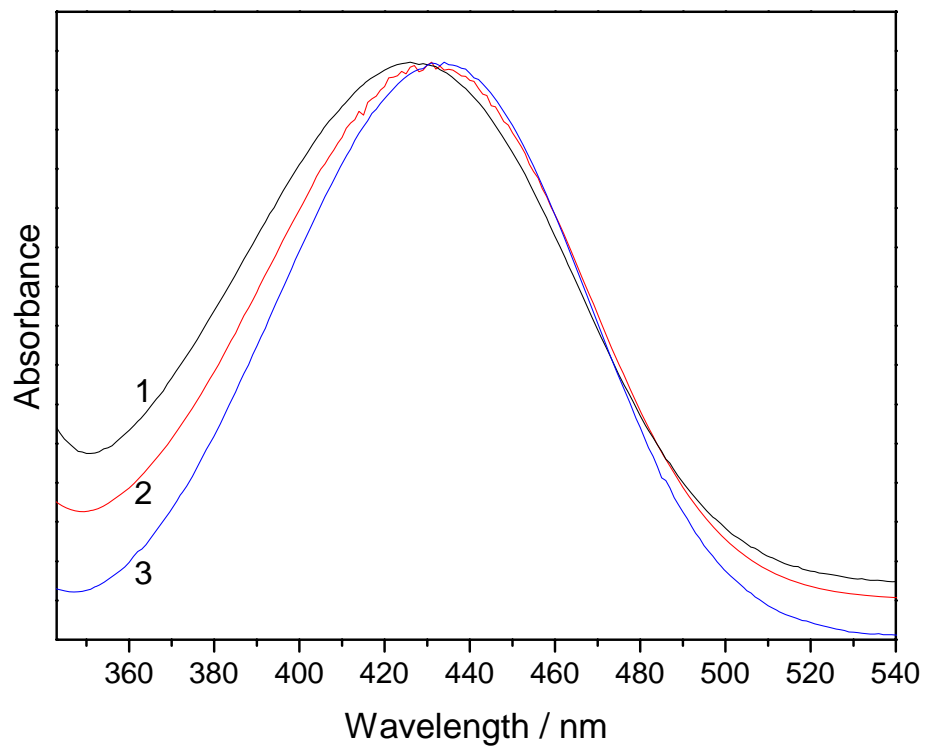

Figure S25. Optical absorption spectra of compound 3a (1) $1 \times 10^{-5} \mathrm{M}$ (black), (2) $1 \times 10^{-4} \mathrm{M}$ (red), (3) $5 \times 10^{-3} \mathrm{M}$ (blue) in 1:1 $\mathrm{PhH} / \mathrm{MeCN}$. 